\title{
Existence criteria for positive solutions of $p$-Laplacian fractional differential equations with derivative terms
}

\author{
Ying Su${ }^{1 *}$, Qing $\mathrm{Li}^{1}$ and Xi-Lan Liu
}

\section{"Correspondence:} suyingxzit@163.com 'Department of Mathematics, Xuzhou Institute of Technology, Xuzhou, 221116, China Full list of author information is available at the end of the article

\begin{abstract}
In the paper, we consider the existence criteria for positive solutions of the nonlinear $p$-Laplacian fractional differential equation whose nonlinearity contains the first-order derivative explicitly

$$
\left\{\begin{array}{l}
\left(\varphi_{p}\left({ }^{C} D^{\alpha} u(t)\right)\right)^{\prime}=\varphi_{p}(\lambda) f\left(t, u(t), u^{\prime}(t)\right), \quad t \in(0,1), \\
k_{0} u(0)-k_{1} u(1)=0, \\
m_{0} u(0)-m_{1} u(1)=0, \\
x^{(r)}(0)=0, \quad r=2,3, \ldots,[\alpha],
\end{array}\right.
$$

where $\varphi_{p}$ is the $p$-Laplacian operator, i.e., $\varphi_{p}(s)=|s|^{p-2} s, p>1$, and $\varphi_{q}=\varphi_{p}^{-1}, \frac{1}{p}+\frac{1}{q}=1$. ${ }^{c} D^{\alpha}$ is the standard Caputo derivative and $f\left(t, u, u^{\prime}\right):[0,1] \times[0, \infty) \times(-\infty,+\infty) \rightarrow$ $[0, \infty)$ satisfies the Carathéodory type condition. The nonlinear alternative of Leray-Schauder type and the fixed-point theorems in Banach space are used to investigate the existence of at least single, twin, triple, $n$ or $2 n-1$ positive solutions for $p$-Laplacian fractional order differential equations. As an application, two examples are given to illustrate our theoretical results.
\end{abstract}

MSC: $34 \mathrm{~A} 08 ; 34 \mathrm{~B} 18 ; 34 \mathrm{~K} 37$

Keywords: existence; positive solution; $p$-Laplacian fractional differential equation; Caputo derivative; fixed-point theorem; Carathéodory type condition

\section{Introduction}

Fractional calculus is a generalization of ordinary differentiation and integration to arbitrary noninteger order. The increasing interest of fractional equations is motivated by their applications in various fields of science such as physics, fluid mechanics, heat conduction in materials with memory, chemistry and engineering. Fractional derivatives and integrals are proved to be more useful for the formulation of certain electrochemical problems than the classical models [1-8]. In consequence, the subject of fractional differential equations is gaining diverse and continuous attention. For more details of some recent theoretical results on fractional differential equations and their applications, we refer the reader to [9-16] and the references therein.

Turbulent flow in a porous medium is a fundamental mechanics problem. For studying this type of problem, Leibenson [17] introduced the following $p$-Laplacian differential

\section{焦 Springer}

(c) 2013 Su et al.; licensee Springer. This is an Open Access article distributed under the terms of the Creative Commons Attribution License (http://creativecommons.org/licenses/by/2.0), which permits unrestricted use, distribution, and reproduction in any medium, provided the original work is properly cited. 
equation:

$$
\left(\varphi_{p}\left(u^{\prime}(t)\right)\right)^{\prime}=f\left(t, u(t), u^{\prime}(t)\right), \quad t \in(0,1)
$$

where $\varphi_{p}(s)=|s|^{p-2} s, p>1$, and $\varphi_{q}=\varphi_{p}^{-1}, \frac{1}{p}+\frac{1}{q}=1$. The study of differential equation (1) is of significance theoretically and practically [7], then many important results relative to differential equation (1) with different boundary value conditions have been obtained. It is quite natural that to investigate arbitrary noninteger order differential equation relative to equation (1).

Motivated by the references [17-19], in this paper, we consider the following $p$-Laplacian fractional differential equations with Caputo fractional derivative:

$$
\left\{\begin{array}{l}
\left(\varphi_{p}\left({ }^{C} D^{\alpha} u(t)\right)\right)^{\prime}=\varphi_{p}(\lambda) f\left(t, u(t), u^{\prime}(t)\right), \quad t \in(0,1), \\
k_{0} u(0)-k_{1} u(1)=0 \\
m_{0} u(0)-m_{1} u(1)=0 \\
x^{(r)}(0)=0, \quad r=2,3, \ldots,[\alpha]
\end{array}\right.
$$

where $\varphi_{p}$ is the $p$-Laplacian operator, i.e., $\varphi_{p}(s)=|s|^{p-2} s, p>1$, and $\varphi_{q}=\varphi_{p}^{-1}, \frac{1}{p}+\frac{1}{q}=1$. ${ }^{C} D^{\alpha}$ is the standard Caputo derivative and $f\left(t, u, u^{\prime}\right):[0,1] \times[0, \infty) \times(-\infty,+\infty) \rightarrow[0, \infty)$ satisfies the Carathéodory type condition. $\alpha>2$ is real and $[\alpha]$ denotes the integer part of the real number $\alpha, \lambda>0, k_{i}, m_{i}(i=0,1)$ are constants satisfying $0<k_{1}<k_{0}$ and $0<m_{1}<m_{0}$. The existence criteria of at least one or two positive solutions are established by using the nonlinear alternative of Leray-Schauder type and the Krasnosel'skii's fixed-point theorem, and the existence of at least $n$ or $2 n-1$ distinct positive solutions are obtained by using of the Leggett-Williams fixed-point theorem, the generalized Avery-Henderson fixed-point theorem as well as the Avery-Peterson fixed-point theorem.

The rest of the paper is organized as follows. In Section 2, we present some basic definitions and several fixed-point theorems. In Section 3, we give and discuss the completely continuous operator of $p$-Laplacian fractional differential equation (2). In Section 4, by using the nonlinear alternative of Leray-Schauder type and the Krasnosel'skii's fixed-point theorem, some new sufficient conditions of the existence of at least one or two positive solutions of $p$-Laplacian fractional differential equation (2) are obtained. In Section 5, the existence criteria for at least three or arbitrary $n$ or $2 n-1$ positive solutions of $p$-Laplacian fractional differential equation (2) are established. In Section 6, we present two examples.

In this study, we assume that $f\left(t, u_{1}, u_{2}\right):[0,1] \times[0, \infty) \times(-\infty,+\infty) \rightarrow[0, \infty)$ satisfies the following conditions of Carathéodory type:

(S1) $f\left(t, u_{1}, u_{2}\right)$ is Lebesgue measurable with respect to $t$ on $[0,1]$;

(S2) for a.e. $t \in[0,1], f(t, \cdot, \cdot)$ is continuous on $[0,1] \times[0, \infty) \times(-\infty,+\infty)$.

\section{Preliminaries}

In this section, we list some basic definitions and the several fixed-point theorems, which help us to better understand our proofs presented in next a few sections. 
Definition 1 [1] Let $\alpha>0$, the fractional integral of order $\alpha$ of function $y:(0, \infty) \rightarrow \mathbb{R}$ is defined by

$$
I^{\alpha} y(t)=\frac{1}{\Gamma(\alpha)} \int_{0}^{t}(t-s)^{\alpha-1} y(s) d s
$$

provided the integral exists.

Definition 2 [1] The Caputo derivative of a function $y:(0, \infty) \rightarrow \mathbb{R}$ is given by

$$
{ }^{C} D^{\alpha} y(t)=\frac{1}{\Gamma(n-\alpha)} \int_{0}^{t} \frac{y^{(n)}(s)}{(t-s)^{\alpha+1-n}} d s
$$

provided that the right side is pointwise defined on $(0, \infty)$, where $n-1<\alpha<n$ and $n$ is a integer.

The Gamma function is given by

$$
\Gamma(\alpha)=\int_{0}^{+\infty} e^{-t} t^{\alpha-1} d t
$$

and the Beta function is given by

$$
B(p, q)=\int_{0}^{1} t^{p-1}(1-t)^{q-1} d t
$$

In addition,

$$
B(p, q)=\frac{\Gamma(p) \Gamma(q)}{\Gamma(p+q)}
$$

The following are two fixed point theorems. The former one is the so-called nonlinear alternative of Leray-Schauder type and the latter one is the Krasnosel'skii's fixed-point theorem $[20,21]$.

Lemma 1 Let $X$ be a Banach space with $C \subset X$ being closed and convex. Assume that $U$ is a relativelyopen subset of $C$ with $0 \in U$ and $A: \bar{U} \rightarrow C$ is a completely continuous operator, then either

(i) A has a fixed point in $\bar{U}$, or

(ii) there exists $u \in \partial U$ and $\gamma_{1}^{*} \in(0,1)$ with $u=\gamma_{1}^{*} A u$.

Lemma 2 Let $P$ be a cone in a Banach space E. Assume $\Omega_{1}$ and $\Omega_{2}$ are open subsets of $E$ with $0 \in \Omega_{1}$ and $\bar{\Omega}_{1} \subset \Omega_{2}$. If $A: P \cap\left(\bar{\Omega}_{2} \backslash \Omega_{1}\right) \rightarrow P$ is a completely continuous operator such that either

(i) $\|A x\| \leq\|x\|, \forall x \in P \cap \partial \Omega_{1}$ and $\|A x\| \geq\|x\|, \forall x \in P \cap \partial \Omega_{2}$, or

(ii) $\|A x\| \geq\|x\|, \forall x \in P \cap \partial \Omega_{1}$ and $\|A x\| \leq\|x\|, \forall x \in P \cap \partial \Omega_{2}$.

Then $A$ has a fixed point in $P \cap\left(\bar{\Omega}_{2} \backslash \Omega_{1}\right)$. 
Assume that $P_{c}=\{u \in P:\|u\|<c\}$ and $P(q, b, d)=\{u \in P: b \leq q(u),\|u\| \leq d\}$, where the map $q$ is a nonnegative continuous concave functional on $P$. The following two theorems are the Leggett and Williams fixed-point theorems [22] and the generalized AveryHenderson fixed-point theorem [23], respectively.

Lemma 3 Suppose that $A: \bar{P}_{c} \rightarrow \bar{P}_{c}$ is completely continuous and there exists a concave positive functional q on P such that $q(u) \leq\|u\|$ for $u \in \bar{P}_{c}$. Suppose that there exist constants $0<a<b<d \leq c$ such that:

(i) $\{u \in P(q, b, d): q(u)>b\} \neq \emptyset$ and $q(T u)>b$ if $u \in P(q, b, d)$;

(ii) $\|T u\|<a$ if $u \in P_{a}$;

(iii) $q(T u)>b$ for $u \in P(q, b, c)$ with $\|T u\|>d$.

Then $A$ has at least three fixed points $u_{1}, u_{2}$ and $u_{3}$ such that

$$
\left\|u_{1}\right\|<a, \quad b<q\left(u_{2}\right) \quad \text { and } \quad u_{3}>a \quad \text { with } \quad q\left(u_{3}\right)<b .
$$

For each $d>0$, let $P(\gamma, d)=\{x \in P: \gamma(x)<d\}$, where $\gamma$ is a nonnegative continuous functional on a cone $P$ of a real Banach space $E$.

Lemma 4 Let $P$ be a cone in a real Banach space E. Let $\alpha, \beta$ and $\gamma$ be increasing, nonnegative continuous functionals on $P$ such that for some $c>0$ and $H>0, \gamma(x) \leq \beta(x) \leq \alpha(x)$ and $\|x\| \leq H \gamma(x)$ for all $x \in \bar{P}(\gamma, c)$. Suppose that there exist positive numbers $a$ and $b$ with $a<b<c$, and $A: \bar{P}(\gamma, c) \rightarrow P$ is a completely continuous operator such that:

(i) $\gamma(A x)<c$ for all $x \in \partial P(\gamma, c)$;

(ii) $\beta(A x)>b$ for all $x \in \partial P(\beta, b)$;

(iii) $P(\alpha, a) \neq \emptyset$ and $\alpha(A x)<a$ for $x \in \partial P(\alpha, a)$.

Then $A$ has at least three fixed points $x_{1}, x_{2}$ and $x_{3}$ belonging to $\bar{P}(\gamma, c)$ such that

$$
0 \leq \alpha\left(x_{1}\right)<a<\alpha\left(x_{2}\right) \text { with } \beta\left(x_{2}\right)<b<\beta\left(x_{3}\right) \text { and } \gamma\left(x_{3}\right)<c
$$

Let $\beta$ and $\phi$ be nonnegative continuous convex functionals on $P, \lambda$ be a nonnegative continuous concave functional on $P$ and $\varphi$ be a nonnegative continuous functional on $P$. We define the following convex sets:

$$
\begin{aligned}
& P(\phi, \lambda, b, d)=\{x \in P: b \leq \lambda(x), \phi(x) \leq d\}, \\
& P(\phi, \beta, \lambda, b, c, d)=\{x \in P: b \leq \lambda(x), \beta(x) \leq c, \phi(x) \leq d\},
\end{aligned}
$$

and

$$
R(\phi, \varphi, a, d)=\{x \in P: a \leq \varphi(x), \phi(x) \leq d\} .
$$

We are ready to recall the Avery-Peterson fixed-point theorem [24].

Lemma 5 Let $P$ be a cone in a real Banach space $E$, and $\beta, \phi, \lambda$ and $\varphi$ be defined as the above. Moreover, $\varphi$ satisfies $\varphi\left(\lambda^{\prime} x\right) \leq \lambda^{\prime} \varphi(x)$ for $0 \leq \lambda^{\prime} \leq 1$ such that for some positive numbers $h$ and $d$,

$$
\lambda(x) \leq \varphi(x) \quad \text { and } \quad\|x\| \leq h \phi(x)
$$


holds for all $x \in \overline{P(\phi, d)}$. Suppose that $A: \overline{P(\phi, d)} \rightarrow \overline{P(\phi, d)}$ is completely continuous and there exist positive real numbers $a, b$, with $a<b$ such that:

(i) $\{x \in P(\phi, \beta, \lambda, b, c, d): \lambda(x)>b\} \neq \emptyset$ and $\lambda(A(x))>b$ for $x \in P(\phi, \beta, \lambda, b, c, d)$;

(ii) $\lambda(A(x))>b$ for $x \in P(\phi, \lambda, b, d)$ with $\beta(A(x))>c$;

(iii) $0 \notin R(\phi, \varphi, a, d)$ and $\lambda(A(x))<$ a for all $x \in R(\phi, \varphi, a, d)$ with $\varphi(x)=a$.

Then $A$ has at least three fixed points $x_{1}, x_{2}, x_{3} \in \overline{P(\phi, d)}$ such that

$$
\begin{aligned}
& \phi\left(x_{i}\right) \leq d \quad \text { for } i=1,2,3, \quad b<\lambda\left(x_{1}\right), \\
& a<\varphi\left(x_{2}\right) \quad \text { and } \lambda\left(x_{2}\right)<b \quad \text { with } \varphi\left(x_{3}\right)<a .
\end{aligned}
$$

\section{Completely continuous operator}

In this section, we firstly present some lemmas, which will be used in our discussions. Then we establish the completely continuous operator for our $p$-Laplacian fractional differential equation, and obtain that solving the solutions of $p$-Laplacian fractional differential equation (2) are equivalent to finding the fixed points of the associated completely continuous operator.

Lemma 6 Let $0<n-1<\alpha<n$, assume that $u \in C^{n}(0,1) \cap L[0,1]$, then the following fractional differential equation:

$$
{ }^{C} D^{\alpha} u(t)=0
$$

has the unique solution

$$
u(t)=\sum_{k=0}^{n-1} \frac{u^{(k)}(0)}{k !} t^{k} .
$$

Proof It follows from Definition 2 that the result is true.

Lemma 7 Assume that $h \in L\left((0,1), \mathbb{R}^{+}\right)$, then the $p$-Laplacian fractional differential equation

$$
\left\{\begin{array}{l}
\left(\varphi_{p}\left({ }^{C} D^{\alpha} u(t)\right)\right)^{\prime}=\varphi_{p}(\lambda) h(t), \quad t \in(0,1) \\
k_{0} u(0)-k_{1} u(1)=0 \\
m_{0} u(0)-m_{1} u(1)=0 \\
u^{(r)}(0)=0, \quad r=2,3, \ldots,[\alpha]
\end{array}\right.
$$

has a unique solution

$$
\begin{aligned}
u(t)= & \frac{m_{1}}{m_{0}-m_{1}} \frac{\lambda}{\Gamma(\alpha-1)}\left(\frac{k_{1}}{k_{0}-k_{1}}+t\right) \int_{0}^{1}(1-\tau)^{\alpha-2} \varphi_{q}\left(\int_{0}^{\tau} h(s) d s\right) d \tau \\
& +\frac{k_{1}}{k_{0}-k_{1}} \frac{\lambda}{\Gamma(\alpha)} \int_{0}^{1}(1-\tau)^{\alpha-1} \varphi_{q}\left(\int_{0}^{\tau} h(s) d s\right) d \tau \\
& +\frac{\lambda}{\Gamma(\alpha)} \int_{0}^{t}(t-\tau)^{\alpha-1} \varphi_{q}\left(\int_{0}^{\tau} h(s) d s\right) d \tau .
\end{aligned}
$$


Proof It follows from (5) that

$$
{ }^{C} D^{\alpha} u(t)=\lambda \varphi_{q}\left(\int_{0}^{t} h(s) d s\right) .
$$

The fractional integral of order $\alpha$ of function $u$ denotes by $I^{\alpha} u$, then

$$
\begin{aligned}
u(t) & =u(0)+u^{\prime}(0) t+\frac{u^{\prime \prime}(0)}{2 !} t^{2}+\cdots+\frac{u^{(n-1)}(0)}{(n-1) !} t^{n-1}+I^{\alpha} \lambda \varphi_{q}\left(\int_{0}^{t} h(s) d s\right) \\
& =u(0)+u^{\prime}(0) t+\frac{\lambda}{\Gamma(\alpha)} \int_{0}^{t}(t-\tau)^{\alpha-1} \varphi_{q}\left(\int_{0}^{\tau} h(s) d s\right) d \tau
\end{aligned}
$$

the latter inequality holds since $u^{(r)}(0)=0, r=2,3, \ldots,[\alpha]$. In addition,

$$
u^{\prime}(t)=u^{\prime}(0)+\frac{\lambda}{\Gamma(\alpha-1)} \int_{0}^{t}(t-\tau)^{\alpha-2} \varphi_{q}\left(\int_{0}^{\tau} h(s) d s\right) d \tau,
$$

hence

$$
\begin{aligned}
& u(1)=u(0)+u^{\prime}(0)+\frac{\lambda}{\Gamma(\alpha)} \int_{0}^{1}(1-\tau)^{\alpha-1} \varphi_{q}\left(\int_{0}^{\tau} h(s) d s\right) d \tau, \\
& u^{\prime}(1)=u^{\prime}(0)+\frac{\lambda}{\Gamma(\alpha-1)} \int_{0}^{1}(1-\tau)^{\alpha-2} \varphi_{q}\left(\int_{0}^{\tau} h(s) d s\right) d \tau .
\end{aligned}
$$

The boundary conditions of (5) reduce to

$$
\begin{aligned}
u(0)= & \frac{k_{1}}{k_{0}-k_{1}} \frac{m_{1}}{m_{0}-m_{1}} \frac{\lambda}{\Gamma(\alpha-1)} \int_{0}^{1}(1-\tau)^{\alpha-2} \varphi_{q}\left(\int_{0}^{\tau} h(s) d s\right) d \tau \\
& +\frac{k_{1}}{k_{0}-k_{1}} \frac{\lambda}{\Gamma(\alpha)} \int_{0}^{1}(1-\tau)^{\alpha-1} \varphi_{q}\left(\int_{0}^{\tau} h(s) d s\right) d \tau
\end{aligned}
$$

and

$$
u^{\prime}(0)=\frac{m_{1}}{m_{0}-m_{1}} \frac{\lambda}{\Gamma(\alpha-1)} \int_{0}^{1}(1-\tau)^{\alpha-2} \varphi_{q}\left(\int_{0}^{\tau} h(s) d s\right) d \tau .
$$

Now, plugging (8) and (9) into (7), then (6) is satisfied.

Suppose that $E_{1}=C^{1}([0,1], \mathbb{R})$, then $E_{1}$ is a Banach space endowed with norm

$$
\|u\|=\sup \left\{\|u\|_{1},\|u\|_{2}\right\}
$$

where

$$
\|u\|_{1}=\sup _{t \in[0,1]}|u(t)|
$$

and

$$
\|u\|_{2}=\sup _{t \in[0,1]}\left|u^{\prime}(t)\right|
$$


The cone $P_{1} \subset E_{1}$ is defined by

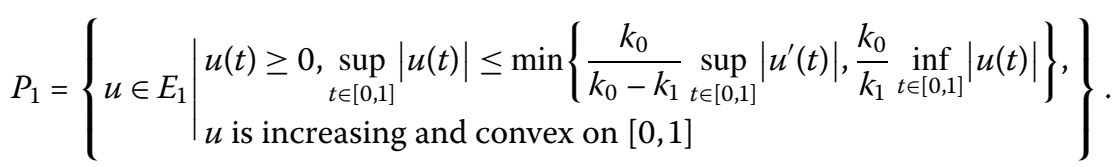

The operator $A: E_{1} \rightarrow E_{1}$ is defined by

$$
\begin{aligned}
(A u)(t)= & \frac{m_{1}}{m_{0}-m_{1}} \frac{\lambda}{\Gamma(\alpha-1)}\left(\frac{k_{1}}{k_{0}-k_{1}}+t\right) \int_{0}^{1}(1-\tau)^{\alpha-2} \varphi_{q}\left(\int_{0}^{\tau} f\left(s, u, u^{\prime}\right) d s\right) d \tau \\
& +\frac{k_{1}}{k_{0}-k_{1}} \frac{\lambda}{\Gamma(\alpha)} \int_{0}^{1}(1-\tau)^{\alpha-1} \varphi_{q}\left(\int_{0}^{\tau} f\left(s, u, u^{\prime}\right) d s\right) d \tau \\
& +\frac{\lambda}{\Gamma(\alpha)} \int_{0}^{t}(t-\tau)^{\alpha-1} \varphi_{q}\left(\int_{0}^{\tau} f\left(s, u, u^{\prime}\right) d s\right) d \tau .
\end{aligned}
$$

Then the solutions of fractional differential equation (2) are the corresponding fixed points of the operator $A$.

Lemma 8 Suppose that conditions (S1) and (S2) are satisfied. For any $t \in[0,1]$ and all $\left(u_{1}, u_{2}\right) \in[0,+\infty) \times(-\infty,+\infty)$, we assume that there exist two nonnegative real-value functions $a_{1}, a_{2} \in L[0,1]$ such that

$$
\int_{0}^{t} f\left(s, u_{1}, u_{2}\right) d s \leq \varphi_{p}\left(a_{1}(t)+a_{2}(t) \max _{t \in[0,1]} u_{1}(t)\right),
$$

or

$$
\int_{0}^{t} f\left(s, u_{1}, u_{2}\right) d s \leq \varphi_{p}\left(a_{1}(t)+a_{2}(t) \max _{t \in[0,1]}\left|u_{2}(t)\right|\right) .
$$

Then the operator $A: P_{1} \rightarrow P_{1}$ is completely continuous.

Proof of Lemma 8 Firstly, we show that $A: P_{1} \rightarrow P_{1}$ is continuous.

Let $u \in P_{1}$, it is obvious that $A u(t) \geq 0$ for arbitrary $t \in[0,1]$. By using the property of the fractional integral and derivative, we obtain that

$$
\begin{aligned}
(A u)^{\prime}(t)= & \frac{m_{1}}{m_{0}-m_{1}} \frac{\lambda}{\Gamma(\alpha-1)} \int_{0}^{1}(1-\tau)^{\alpha-2} \varphi_{q}\left(\int_{0}^{\tau} f\left(s, u, u^{\prime}\right) d s\right) d \tau \\
& +\frac{\lambda}{\Gamma(\alpha-1)} \int_{0}^{t}(t-\tau)^{\alpha-2} \varphi_{q}\left(\int_{0}^{\tau} f\left(s, u, u^{\prime}\right) d s\right) d \tau \geq 0,
\end{aligned}
$$

and

$$
(A u)^{\prime \prime}(t)=\frac{\lambda}{\Gamma(\alpha-2)} \int_{0}^{t}(t-\tau)^{\alpha-3} \varphi_{q}\left(\int_{0}^{\tau} f\left(s, u, u^{\prime}\right) d s\right) d \tau \geq 0 .
$$

Since $A x$ is nonnegative, increasing and convex on $[0,1]$, we have

$$
\begin{aligned}
& \sup _{t \in[0,1]}|(A u)(t)|=\max _{t \in[0,1]}(A u)(t)=(A u)(1), \\
& \sup _{t \in[0,1]}\left|(A u)^{\prime}(t)\right|=\max _{t \in[0,1]}(A u)^{\prime}(t)=(A u)^{\prime}(1),
\end{aligned}
$$


and

$$
\inf _{t \in[0,1]}|(A u)(t)|=(A u)(0), \quad \inf _{t \in[0,1]}\left|(A u)^{\prime}(t)\right|=(A u)^{\prime}(0) .
$$

Moreover,

$$
\sup _{t \in[0,1]}|(A u)(t)|=(A u)(1)=\frac{k_{0}}{k_{1}}(A u)(0)=\frac{k_{0}}{k_{1}} \inf _{t \in[0,1]}|(A u)(t)|,
$$

and

$$
\begin{aligned}
\sup _{t \in[0,1]}|(A u)(t)| & \\
= & (A u)(1) \\
= & \frac{m_{1}}{m_{0}-m_{1}} \frac{k_{0}}{k_{0}-k_{1}} \frac{\lambda}{\Gamma(\alpha-1)} \int_{0}^{1}(1-\tau)^{\alpha-2} \varphi_{q}\left(\int_{0}^{\tau} f\left(s, u, u^{\prime}\right) d s\right) d \tau \\
& +\frac{k_{0}}{k_{0}-k_{1}} \frac{\lambda}{\Gamma(\alpha)} \int_{0}^{1}(1-\tau)^{\alpha-1} \varphi_{q}\left(\int_{0}^{\tau} f\left(s, u, u^{\prime}\right) d s\right) d \tau \\
\leq & \frac{m_{1}}{m_{0}-m_{1}} \frac{k_{0}}{k_{0}-k_{1}} \frac{\lambda}{\Gamma(\alpha-1)} \int_{0}^{1}(1-\tau)^{\alpha-2} \varphi_{q}\left(\int_{0}^{\tau} f\left(s, u, u^{\prime}\right) d s\right) d \tau \\
& +\frac{k_{0}}{k_{0}-k_{1}} \frac{\lambda}{\Gamma(\alpha-1)} \int_{0}^{1}(1-\tau)^{\alpha-2} \varphi_{q}\left(\int_{0}^{\tau} f\left(s, u, u^{\prime}\right) d s\right) d \tau \\
= & \frac{k_{0}}{k_{0}-k_{1}} \frac{m_{0}}{m_{0}-m_{1}} \frac{\lambda}{\Gamma(\alpha-1)} \int_{0}^{1}(1-\tau)^{\alpha-2} \varphi_{q}\left(\int_{0}^{\tau} f\left(s, u, u^{\prime}\right) d s\right) d \tau \\
= & \frac{k_{0}}{k_{0}-k_{1}}(A u)^{\prime}(1)=\frac{k_{0}}{k_{0}-k_{1}} \sup _{t \in[0,1]}\left|(A u)^{\prime}(t)\right| .
\end{aligned}
$$

Therefore,

$$
\sup _{t \in[0,1]}|(A u)(t)| \leq\left\{\frac{k_{0}}{k_{1}} \inf _{t \in[0,1]}|(A u)(t)|, \frac{k_{0}}{k_{0}-k_{1}} \sup _{t \in[0,1]}\left|(A u)^{\prime}(t)\right|\right\},
$$

which implies that $A: P_{1} \rightarrow P_{1}$.

Suppose that $\left\{u_{n}\right\}_{n=1}^{\infty} \subset \bar{P}_{1}, n=1,2, \ldots$, and $u_{n}$ uniformly converges to $u$ on $[0,1]$, that is

$$
\lim _{n \rightarrow \infty}\left\|u_{n}-u\right\|=0
$$

So, we have

$$
\lim _{n \rightarrow \infty}\left\|u_{n}-u\right\|_{1}=0 \quad \text { and } \quad \lim _{n \rightarrow \infty}\left\|u_{n}-u\right\|_{2}=0
$$

which implies that

$$
\lim _{n \rightarrow \infty} u_{n}(t)=u(t) \quad \text { and } \quad \lim _{n \rightarrow \infty} u_{n}^{\prime}(t)=u^{\prime}(t), \quad t \in[0,1] .
$$

Then (S1) and the continuous of $\varphi_{q}$ imply that

$$
\lim _{n \rightarrow \infty} \varphi_{q}\left(f\left(t, u_{n}(t), u_{n}^{\prime}(t)\right)\right)=\varphi_{q}\left(f\left(t, u(t), u^{\prime}(t)\right)\right), \quad t \in[0,1]
$$


then, we obtain

$$
\begin{aligned}
\left|A u_{n}(t)-A u(t)\right| & \\
\leq & \frac{m_{1}}{m_{0}-m_{1}} \frac{\lambda}{\Gamma(\alpha-1)}\left(\frac{k_{1}}{k_{0}-k_{1}}+t\right) \\
& \times\left|\int_{0}^{1}(1-\tau)^{\alpha-2}\left(\varphi_{q}\left(\int_{0}^{\tau} f\left(s, u_{n}, u_{n}^{\prime}\right) d s\right)-\varphi_{q}\left(\int_{0}^{\tau} f\left(s, u, u^{\prime}\right) d s\right)\right) d \tau\right| \\
& +\mid \frac{k_{1}}{k_{0}-k_{1}} \frac{\lambda}{\Gamma(\alpha)} \int_{0}^{1}(1-\tau)^{\alpha-1}\left(\varphi_{q}\left(\int_{0}^{\tau} f\left(s, u_{n}, u_{n}^{\prime}\right) d s\right)\right. \\
& \left.-\varphi_{q}\left(\int_{0}^{\tau} f\left(s, u, u^{\prime}\right) d s\right)\right) d \tau \mid \\
& +\left|\frac{\lambda}{\Gamma(\alpha)} \int_{0}^{1}(t-\tau)^{\alpha-1}\left(\varphi_{q}\left(\int_{0}^{\tau} f\left(s, u_{n}, u_{n}^{\prime}\right) d s\right)-\varphi_{q}\left(\int_{0}^{\tau} f\left(s, u, u^{\prime}\right) d s\right)\right) d \tau\right| \\
\rightarrow & 0 \text { as } n \rightarrow \infty,
\end{aligned}
$$

and

$$
\begin{aligned}
& \left|A^{\prime} u_{n}(t)-A^{\prime} u(t)\right| \\
& \leq \mid \frac{m_{1}}{m_{0}-m_{1}} \frac{\lambda}{\Gamma(\alpha-1)} \int_{0}^{1}(1-\tau)^{\alpha-2}\left(\varphi_{q}\left(\int_{0}^{\tau} f\left(s, u_{n}, u_{n}^{\prime}\right) d s\right)\right. \\
& \left.\quad-\varphi_{q}\left(\int_{0}^{\tau} f\left(s, u, u^{\prime}\right) d s\right)\right) d \tau \mid \\
& \quad+\left|\frac{\lambda}{\Gamma(\alpha-1)} \int_{0}^{1}(t-\tau)^{\alpha-2}\left(\varphi_{q}\left(\int_{0}^{\tau} f\left(s, u_{n}, u_{n}^{\prime}\right) d s\right)-\varphi_{q}\left(\int_{0}^{\tau} f\left(s, u, u^{\prime}\right) d s\right)\right) d \tau\right| \\
& \quad \rightarrow 0 \text { as } n \rightarrow \infty .
\end{aligned}
$$

By virtue of (14) and (15), we have

$$
\left\|\left(A u_{n}\right)(t)-(A u)(t)\right\| \rightarrow 0 \quad \text { as } n \rightarrow \infty,
$$

which means that $A$ is continuous.

Secondly, we show that $A$ maps bounded sets into bounded sets in $P_{1}$. It suffices to show that for any $\eta>0$, there is a positive constant $l>0$ such that for each $u \in B_{\eta}=\left\{u \in P_{1}\right.$ : $\|u\| \leq \eta\}$, we have $\|A u\| \leq l$.

Let

$$
\begin{aligned}
l & =\frac{k_{0}}{k_{0}-k_{1}} \frac{m_{0}}{m_{0}-m_{1}} \frac{\lambda}{\Gamma(\alpha-1)}\left(\int_{0}^{1}(1-\tau)^{\alpha-2} a_{1}(\tau) d \tau+\eta \int_{0}^{1}(1-\tau)^{\alpha-2} a_{2}(\tau) d \tau\right) \\
& >0 .
\end{aligned}
$$

According to (11) and (13), we have

$$
\begin{aligned}
|A u(t)| & \leq \frac{k_{0}}{k_{0}-k_{1}} \frac{m_{0}}{m_{0}-m_{1}} \frac{\lambda}{\Gamma(\alpha-1)} \int_{0}^{1}(1-\tau)^{\alpha-2} \varphi_{q}\left(\int_{0}^{\tau} f\left(s, u, u^{\prime}\right) d s\right) d \tau \\
& \leq \frac{k_{0}}{k_{0}-k_{1}} \frac{m_{0}}{m_{0}-m_{1}} \frac{\lambda}{\Gamma(\alpha-1)} \int_{0}^{1}(1-\tau)^{\alpha-2}\left(a_{1}(\tau)+a_{2}(\tau) \max _{t \in[0,1]} u(t)\right) d \tau
\end{aligned}
$$




$$
\begin{aligned}
& \leq \frac{k_{0}}{k_{0}-k_{1}} \frac{m_{0}}{m_{0}-m_{1}} \frac{\lambda}{\Gamma(\alpha-1)}\left(\int_{0}^{1}(1-\tau)^{\alpha-2} a_{1}(\tau) d \tau+\eta \int_{0}^{1}(1-\tau)^{\alpha-2} a_{2}(\tau) d \tau\right) \\
& =l
\end{aligned}
$$

Using (12) and (13) yields

$$
\begin{aligned}
|A u(t)| & \leq \frac{k_{0}}{k_{0}-k_{1}} \frac{m_{0}}{m_{0}-m_{1}} \frac{\lambda}{\Gamma(\alpha-1)} \int_{0}^{1}(1-\tau)^{\alpha-2}\left(a_{1}(\tau)+a_{2}(\tau) \max _{t \in[0,1]}\left|u^{\prime}(t)\right|\right) d \tau \\
& \leq \frac{k_{0}}{k_{0}-k_{1}} \frac{m_{0}}{m_{0}-m_{1}} \frac{\lambda}{\Gamma(\alpha-1)}\left(\int_{0}^{1}(1-\tau)^{\alpha-2} a_{1}(\tau) d \tau+\eta \int_{0}^{1}(1-\tau)^{\alpha-2} a_{2}(\tau) d \tau\right) \\
& =l
\end{aligned}
$$

In addition, using (11) and (12), we also have

$$
\begin{aligned}
\left|(A u)^{\prime}(t)\right| \leq & \frac{m_{0}}{m_{0}-m_{1}} \frac{\lambda}{\Gamma(\alpha-1)} \int_{0}^{1}(1-\tau)^{\alpha-2} \varphi_{q}\left(\int_{0}^{\tau} f\left(s, u, u^{\prime}\right) d s\right) d \tau \\
\leq & \frac{k_{0}}{k_{0}-k_{1}} \frac{m_{0}}{m_{0}-m_{1}} \frac{\lambda}{\Gamma(\alpha-1)} \\
& \times\left(\int_{0}^{1}(1-\tau)^{\alpha-2} a_{1}(\tau) d \tau+\eta \int_{0}^{1}(1-\tau)^{\alpha-2} a_{2}(\tau) d \tau\right) \\
= & l .
\end{aligned}
$$

Hence, we have $\|A u\| \leq l$.

Thirdly, we consider that $A$ maps bounded sets into equicontinuous sets of $P_{1}$. Since $(t-s)^{\alpha-1}$ and $t$ are uniformly continuous on $[0,1]$, then for any $\varepsilon>0$, there exists $\delta>0$, whenever $\left|t_{1}-t_{2}\right|<\delta$, we have

$$
\left|\left(t_{1}-s\right)^{\alpha-1}-\left(t_{2}-s\right)^{\alpha-1}\right|<\frac{\Gamma(\alpha) \varepsilon}{2 \lambda\left[a_{1}(\tau)+\eta a_{2}(\tau)\right]},
$$

we also obtain

$$
\left|t_{1}-t_{2}\right|<\frac{\varepsilon}{2 \int_{0}^{1}(1-\tau)^{\alpha-2}\left[a_{1}(\tau)+\eta a_{2}(\tau)\right] d \tau} .
$$

For convenience, we assume $t_{1}<t_{2}$. For any $u \in B_{\eta}$, according to (11) and (12), we get

$$
\begin{aligned}
\left|A u\left(t_{2}\right)-A u\left(t_{1}\right)\right| \leq & \left|\left(t_{1}-t_{2}\right) \int_{0}^{1}(1-\tau)^{\alpha-2} \varphi_{q}\left(\int_{0}^{\tau} f\left(s, u, u^{\prime}\right) d s\right) d \tau\right| \\
& +\left|\frac{\lambda}{\Gamma(\alpha)} \int_{0}^{1}\left[\left(t_{1}-\tau\right)^{\alpha-1}-\left(t_{2}-\tau\right)^{\alpha-1}\right] \varphi_{q}\left(\int_{0}^{\tau} f\left(s, u, u^{\prime}\right) d s\right) d \tau\right| \\
& <\left|\left(t_{1}-t_{2}\right) \int_{0}^{1}(1-\tau)^{\alpha-2}\left[a_{1}(\tau)+\eta a_{2}(\tau)\right] d \tau\right| \\
& +\left|\frac{\lambda}{\Gamma(\alpha)} \int_{0}^{1}\left[\left(t_{1}-\tau\right)^{\alpha-1}-\left(t_{2}-\tau\right)^{\alpha-1}\right]\left[a_{1}(\tau)+\eta a_{2}(\tau)\right] d \tau\right| \\
< & \varepsilon,
\end{aligned}
$$


and

$$
\begin{aligned}
& \left|(A u)^{\prime}\left(t_{2}\right)-(A u)^{\prime}\left(t_{1}\right)\right| \\
& \quad \leq\left|\frac{\lambda}{\Gamma(\alpha-1)} \int_{0}^{1}\left[\left(t_{1}-\tau\right)^{\alpha-2}-\left(t_{2}-\tau\right)^{\alpha-2}\right] \varphi_{q}\left(\int_{0}^{\tau} f\left(s, u, u^{\prime}\right) d s\right) d \tau\right| \\
& \quad \leq \frac{\lambda}{\Gamma(\alpha-1)} \int_{0}^{1}\left|\left[\left(t_{1}-\tau\right)^{\alpha-2}-\left(t_{2}-\tau\right)^{\alpha-2}\right]\right|\left[a_{1}(s)+\eta a_{2}(s)\right] d s \\
& \quad<\frac{\Gamma(\alpha)}{2 \Gamma(\alpha-1)} \varepsilon .
\end{aligned}
$$

Consequently, we obtain

$$
\left\|A u\left(t_{2}\right)-A u\left(t_{1}\right)\right\|<\min \left\{\frac{\Gamma(\alpha)}{2 \Gamma(\alpha-1)}, 1\right\} \varepsilon,
$$

which implies that the family of functions $\left\{A u: u \in B_{\eta}\right\}$ is equicontinuous. It follows from the virtue of the Arzela-Ascoli theorem that the operator $A: P_{1} \rightarrow P_{1}$ is completely continuous.

Remark 1 If $f\left(t, u_{1}, u_{2}\right):[0,1] \times[0, \infty) \times(-\infty,+\infty) \rightarrow[0, \infty)$ is continuous, we can obtain that $A: P_{1} \rightarrow P_{1}$ is completely continuous by using a similar argument as the above.

\section{Existence of one or two solutions}

In this section, we discuss the existence of single or twin positive solutions to problem (2).

Theorem 1 Assume that all assumptions of Lemma 8 and

$$
\lambda \int_{0}^{1}(1-\tau)^{\alpha-2} a_{2}(\tau) d \tau<\frac{\left(k_{0}-k_{1}\right)\left(m_{0}-m_{1}\right) \Gamma(\alpha-1)}{k_{0} m_{0}}
$$

hold, then the fractional differential equation (2) has at least one positive solution.

Proof of Theorem 1 Let

$$
U=\left\{u \in P_{1}:\|u\|<r\right\}
$$

where

$$
r=\frac{\frac{k_{0}}{k_{0}-k_{1}} \frac{m_{0}}{m_{0}-m_{1}} \frac{\lambda}{\Gamma(\alpha-1)} \int_{0}^{1}(1-\tau)^{\alpha-2} a_{1}(\tau) d \tau}{1-\frac{k_{0}}{k_{0}-k_{1}} \frac{m_{0}}{m_{0}-m_{1}} \frac{\lambda}{\Gamma(\alpha-1)} \int_{0}^{1}(1-\tau)^{\alpha-2} a_{2}(\tau) d \tau}>0 .
$$

Assume that there exist $u \in P_{1}$ and $\gamma_{1}^{*} \in(0,1)$ such that $u=\gamma_{1}^{*} A u$. Then we find

$$
\begin{aligned}
u(t)= & \gamma_{1}^{*} A u \leq \gamma_{1}^{*} A u(1) \\
\leq & \gamma_{1}^{*} \frac{k_{0}}{k_{0}-k_{1}} \frac{m_{0}}{m_{0}-m_{1}} \frac{\lambda}{\Gamma(\alpha-1)} \\
& \times \int_{0}^{1}(1-\tau)^{\alpha-2}\left(a_{1}(\tau)+a_{2}(\tau) \max \left\{\max _{t \in[0,1]} u(t), \max _{t \in[0,1]}\left|u^{\prime}(t)\right|\right\}\right) d \tau
\end{aligned}
$$




$$
\begin{aligned}
\leq & \gamma_{1}^{*} \frac{k_{0}}{k_{0}-k_{1}} \frac{m_{0}}{m_{0}-m_{1}} \frac{\lambda}{\Gamma(\alpha-1)} \\
& \times\left(\int_{0}^{1}(1-\tau)^{\alpha-2} a_{1}(\tau) d \tau+\|u\| \int_{0}^{1}(1-\tau)^{\alpha-2} a_{2}(\tau) d \tau\right),
\end{aligned}
$$

and

$$
\begin{aligned}
\left|u^{\prime}(t)\right|= & \left|\gamma_{1}^{*}(A u)^{\prime}(t)\right| \\
= & \left|\gamma_{1}^{*} \frac{m_{0}}{m_{0}-m_{1}} \frac{\lambda}{\Gamma(\alpha-1)} \int_{0}^{1}(1-\tau)^{\alpha-2} \varphi_{q}\left(\int_{0}^{\tau} f\left(s, u, u^{\prime}\right) d s\right) d \tau\right| \\
\leq & \gamma_{1}^{*} \frac{k_{0}}{k_{0}-k_{1}} \frac{m_{0}}{m_{0}-m_{1}} \frac{\lambda}{\Gamma(\alpha-1)} \\
& \times\left(\int_{0}^{1}(1-\tau)^{\alpha-2} a_{1}(\tau) d \tau+\|u\| \int_{0}^{1}(1-\tau)^{\alpha-2} a_{2}(\tau) d \tau\right) .
\end{aligned}
$$

Thus, we have

$$
\begin{aligned}
\|u\|< & \frac{k_{0}}{k_{0}-k_{1}} \frac{m_{0}}{m_{0}-m_{1}} \frac{\lambda}{\Gamma(\alpha-1)} \gamma_{1}^{*} \\
& \quad \times\left(\int_{0}^{1}(1-\tau)^{\alpha-2} a_{1}(\tau) d \tau+r \int_{0}^{1}(1-\tau)^{\alpha-2} a_{2}(\tau) d \tau\right) \\
= & \gamma_{1}^{*} r,
\end{aligned}
$$

which means that $u \notin \partial U$ and $\|u\| \neq r$.

By virtue of Lemma 1, we conclude that the fractional differential equation (2) has at least one positive solution.

Remark 2 'All assumptions of Lemma 8 hold' can be replaced by ' $f\left(t, u_{1}, u_{2}\right):[0,1] \times$ $[0, \infty) \times(-\infty,+\infty) \rightarrow[0, \infty)$ is continuous' in Theorem 1 .

Theorem 2 Assume that all assumptions of Lemma 8 and the following conditions hold:

(i) there exists a constant $m>0$ such that $f\left(t, u_{1}, u_{2}\right) \leq \varphi_{p}\left(m \Lambda_{1}\right)$ for $\left(t, u_{1}, u_{2}\right) \in[0,1] \times[0, m] \times[-m, m]$, where $\Lambda_{1}=\left(\frac{k_{0}}{k_{0}-k_{1}} \frac{m_{0}}{m_{0}-m_{1}} \frac{\lambda \Gamma(q)}{\Gamma(\alpha+q-1)}\right)^{-1} ;$

(ii) there exists a constant $e>0$ such that $f\left(t, u_{1}, u_{2}\right) \geq \varphi_{p}\left(e \Lambda_{2}\right)$ for $\left(t, u_{1}, u_{2}\right) \in[0,1] \times[0, e] \times[-e, e]$, where $\Lambda_{2}=\left(\frac{m_{0}}{m_{0}-m_{1}} \frac{\lambda \Gamma(q)}{\Gamma(\alpha+q-1)}\right)^{-1}$, and $m \neq e$.

Then the fractional differential equation (2) has at least one positive solution $u$ such that $\|u\|$ lies between $m$ and $e$.

Proof of Theorem 2 Without loss of generality, we assume that $m<e$.

Let

$$
\Omega_{m}=\left\{u \in E_{1}:\|u\|<m\right\} .
$$

For any $u \in P_{1} \cap \partial \Omega_{m}$, there is

$$
\max _{t \in[0,1]}\left|u^{\prime}(t)\right| \leq\|u\|<m \text { and } \max _{t \in[0,1]} u(t) \leq m
$$


It follows from condition (i) that

$$
\begin{aligned}
\|A u\| & =\max \left\{\|A u\|_{1},\|A u\|_{2}\right\} \\
& \leq \frac{k_{0}}{k_{0}-k_{1}} \frac{m_{0}}{m_{0}-m_{1}} \frac{\lambda}{\Gamma(\alpha-1)} \int_{0}^{1}(1-\tau)^{\alpha-2} \varphi_{q}\left(\int_{0}^{\tau} f\left(s, u, u^{\prime}\right) d s\right) d \tau \\
& <m \Lambda_{1} \frac{k_{0}}{k_{0}-k_{1}} \frac{m_{0}}{m_{0}-m_{1}} \frac{\lambda}{\Gamma(\alpha-1)} \int_{0}^{1}(1-\tau)^{\alpha-2} \tau^{q-1} d \tau \\
& <m \Lambda_{1} \frac{k_{0}}{k_{0}-k_{1}} \frac{m_{0}}{m_{0}-m_{1}} \frac{\lambda \Gamma(q)}{\Gamma(\alpha+q-1)} \\
& \leq m,
\end{aligned}
$$

which implies that

$$
\|A u\| \leq\|u\| \quad \text { for } u \in P_{1} \cap \partial \Omega_{m} .
$$

We define

$$
\Omega_{e}=\left\{u \in E_{1}:\|u\|<e\right\}
$$

for arbitrary $u \in P_{1} \cap \partial \Omega_{e}$, and find

$$
\max _{t \in[0,1]}\left|u^{\prime}(t)\right| \leq\|u\|<e \quad \text { and } \quad \max _{t \in[0,1]} u(t) \leq e .
$$

On the other hand, it follows from condition (ii) that

$$
\begin{aligned}
\|A u\| & \geq\left|\max _{t \in[0,1]}(A u)^{\prime}(t)\right| \\
& =\frac{m_{0}}{m_{0}-m_{1}} \frac{\lambda}{\Gamma(\alpha-1)} \int_{0}^{1}(1-\tau)^{\alpha-2} \varphi_{q}\left(\int_{0}^{\tau} f\left(s, u, u^{\prime}\right) d s\right) d \tau \\
& \geq e \Lambda_{2} \frac{m_{0}}{m_{0}-m_{1}} \frac{\lambda}{\Gamma(\alpha-1)} \int_{0}^{1}(1-\tau)^{\alpha-2} \tau^{q-1} d \tau \\
& \geq e \Lambda_{2} \frac{m_{0}}{m_{0}-m_{1}} \frac{\lambda \Gamma(q)}{\Gamma(\alpha+q-1)}=e,
\end{aligned}
$$

which implies that

$$
\|A u\| \geq\|u\| \quad \text { for } u \in P_{1} \cap \partial \Omega_{e} .
$$

By using (18) and (20), it follows from Lemma 2 that the fractional differential equation (2) has a positive solution $u$ in $P_{1} \cap\left(\bar{\Omega}_{e} \backslash \Omega_{m}\right)$.

Let

$$
\begin{aligned}
& f^{0}=\lim _{\left(u_{1}, u_{2}\right) \rightarrow(0,0)} \sup _{t \in[0,1]} \frac{f\left(t, u_{1}, u_{2}\right)}{\varphi_{p}\left(\left|u_{2}\right|\right)} \quad \text { for } u_{1}, u_{2} \in P_{1}, \\
& f_{\infty}=\lim _{u_{1}+\left|u_{2}\right| \rightarrow \infty} \inf _{t \in[0,1]} \frac{f\left(t, u_{1}, u_{2}\right)}{\varphi_{p}\left(u_{1}+\left|u_{2}\right|\right)} \quad \text { for } u_{1}, u_{2} \in P_{1}, \\
& f_{0}=\lim _{\left(u_{1}, u_{2}\right) \rightarrow(0,0)} \inf _{t \in[0,1]} \frac{f\left(t, u_{1}, u_{2}\right)}{\varphi_{p}\left(u_{1}+\left|u_{2}\right|\right)} \quad \text { for } u_{1}, u_{2} \in P_{1},
\end{aligned}
$$


and

$$
f^{\infty}=\lim _{\left(u_{1}, u_{2}\right) \rightarrow(\infty, \infty)} \sup _{t \in[0,1]} \frac{f\left(t, u_{1}, u_{2}\right)}{\varphi_{p}\left(\left|u_{2}\right|\right)} \quad \text { for } u_{1}, u_{2} \in P_{1}
$$

Now, we have the following two results.

Theorem 3 Assume that all assumptions of Lemma 8 hold. In addition, when $f^{0} \in$ $\left[0, \varphi_{p}\left(\Lambda_{1}\right)\right)$ and $f_{\infty} \in\left(\varphi_{p}\left(\Lambda_{2}\right), \infty\right) \cup\{\infty\}$ hold too, then the fractional differential equation (2) has at least one positive solution.

Proof of Theorem 3 It is easy to obtain that

$$
\sup _{t \in[0,1]} u(t) \leq \frac{k_{0}}{k_{0}-k_{1}} \sup _{t \in[0,1]}\left|u^{\prime}(t)\right|
$$

then, according to the assumption $f^{0}<\varphi_{p}\left(\Lambda_{1}\right)$, there exists a sufficiently small $m>0$ such that

$$
f\left(t, u, u^{\prime}\right) \leq \varphi_{p}\left(\Lambda_{1}\left|u^{\prime}\right|\right) \leq \varphi_{p}\left(\Lambda_{1} m\right) \quad \text { for }\left(t, u, u^{\prime}\right) \in[0,1] \times\left[0, \frac{k_{0}}{k_{0}-k_{1}} m\right] \times[-m, m]
$$

we can obtain (17) is true. That is, if we let

$$
\Omega_{m^{*}}=\left\{u \in E_{1}:\|u\|<\frac{k_{0}}{k_{0}-k_{1}} m\right\}
$$

then

$$
\|A u\| \leq\|u\| \quad \text { for } u \in P_{1} \cap \partial \Omega_{m^{*}}
$$

It follows from $f_{\infty}>\Lambda_{2}$ that there exists an $H>\frac{2 m k_{0}}{k_{0}-k_{1}}$ such that

$$
f\left(t, u, u^{\prime}\right) \geq \varphi_{p}\left(\Lambda_{2}\left(u+\left|u^{\prime}\right|\right)\right) \geq \varphi_{p}\left(\Lambda_{2}\|u\|\right),
$$

where $t \in[0,1]$ and $u+\left|u^{\prime}\right| \geq H$.

Set

$$
\Omega_{H}=\left\{u \in E_{1}: u+\left|u^{\prime}\right|<H\right\},
$$

then we see that $\bar{\Omega}_{m^{*}} \subset \Omega_{H}$.

For any $u \in P_{1} \cap \partial \Omega_{H}$, we have $u+\left|u^{\prime}\right|=H$. Equation (21) gives

$$
\begin{aligned}
\|A u\| & \geq\left|\max _{t \in[0,1]}(A u)^{\prime}(t)\right| \\
& =\frac{m_{0}}{m_{0}-m_{1}} \frac{\lambda}{\Gamma(\alpha-1)} \int_{0}^{1}(1-\tau)^{\alpha-2} \varphi_{q}\left(\int_{0}^{\tau} f\left(s, u, u^{\prime}\right) d s\right) d \tau \\
& \geq \Lambda_{2}\|u\| \frac{m_{0}}{m_{0}-m_{1}} \frac{\lambda}{\Gamma(\alpha-1)} \int_{0}^{1}(1-\tau)^{\alpha-2} t^{q-1} d \tau
\end{aligned}
$$




$$
\begin{aligned}
& \geq \Lambda_{2}\|u\| \frac{m_{0}}{m_{0}-m_{1}} \frac{\lambda \Gamma(q)}{\Gamma(\alpha+q-1)} \\
& =\|u\| .
\end{aligned}
$$

Consequently, it follows from the virtue of Lemma 2 that the fractional differential equation (2) has a positive solution $u$ in $P_{1} \cap\left(\bar{\Omega}_{H} \backslash \Omega_{m^{*}}\right)$.

Theorem 4 Assume that all assumptions of Lemma 8 hold. In addition, $f_{0} \in\left(\varphi_{p}\left(\Lambda_{2}\right), \infty\right) \cup$ $\{\infty\}$ and $f^{\infty} \in\left[0, \varphi_{p}\left(\Lambda_{1}\right)\right)$ are satisfied. Then the fractional differential equation (2) has at least one positive solution.

Proof of Theorem 4 It follows from $f_{0}>\varphi_{p}\left(\Lambda_{2}\right)$ that there exists a sufficiently small $e>0$ such that

$$
\begin{aligned}
f\left(t, u, u^{\prime}\right) & \geq \varphi_{p}\left(\Lambda_{2}\left(u+\left|u^{\prime}\right|\right)\right) \\
& \geq \varphi_{p}\left(\Lambda_{2} \max \left\{\|u\|_{1},\|u\|_{2}\right\}\right) \quad \text { for }\left(t, u, u^{\prime}\right) \in[0,1] \times\left[0, \frac{k_{0}}{k_{0}-k_{1}} e\right] \times[-e, e] .
\end{aligned}
$$

When $\left(t, u, u^{\prime}\right) \in[0,1] \times\left[0, \frac{k_{0}}{k_{0}-k_{1}} e\right] \times[-e, e]$, we get

$$
\begin{aligned}
f\left(t, u, u^{\prime}\right) & \geq \varphi_{p}\left(\Lambda_{2} \max \left\{\|u\|_{1},\|u\|_{2}\right\}\right) \\
& =\varphi_{p}\left(\Lambda_{2} e\right)
\end{aligned}
$$

we can obtain (19). Take

$$
\Omega_{e^{*}}=\left\{u \in E_{1}:\|u\|<\frac{k_{0}}{k_{0}-k_{1}} e\right\} .
$$

Then

$$
\|A u\| \geq\|u\| \quad \text { for } u \in P_{1} \cap \partial \Omega_{e^{*}} .
$$

Let $\varepsilon_{1}=\Lambda_{1}-f^{\infty}(>0)$. Since $f^{\infty}<\varphi_{p}\left(\Lambda_{1}\right)$, there exists a $p_{1}\left(>\frac{k_{0}}{k_{0}-k_{1}} e\right)$ such that

$$
f\left(t, u, u^{\prime}\right) \leq \varphi_{p}\left(\left(\varepsilon_{1}+f^{\infty}\right)\left|u^{\prime}\right|\right)=\varphi_{p}\left(\Lambda_{1}\left|u^{\prime}\right|\right),
$$

where $\left(t, u, u^{\prime}\right) \in[0,1] \times\left[\frac{k_{0}}{k_{0}-k_{1}} p_{1}, \infty\right) \times\left(-\infty,-p_{1}\right] \cup\left[p_{1},+\infty\right)$.

Note that

$$
f \in C([0,1] \times[0, \infty) \times(-\infty, \infty),[0, \infty))
$$

So there exists a $C_{4}>0$ such that

$$
f\left(t, u, u^{\prime}\right) \leq \varphi_{p}\left(C_{4}\right) \quad \text { for }\left(t, u, u^{\prime}\right) \in[0,1] \times\left[0, \frac{k_{0}}{k_{0}-k_{1}} p_{1}\right] \times\left[-p_{1}, p_{1}\right]
$$

Equations (22) and (23) reduce to

$$
f\left(t, u, u^{\prime}\right) \leq \max \left\{\varphi_{p}\left(C_{4}\right), \varphi_{p}\left(\Lambda_{1}\left|u^{\prime}\right|\right)\right\} \quad \text { for }\left(t, u, u^{\prime}\right) \in[0,1] \times[0, \infty) \times(-\infty, \infty) .
$$


Let

$$
p_{2}^{*}>\max \left\{C_{4} / \Lambda_{1}, \frac{2 k_{0}}{k_{0}-k_{1}} e\right\}
$$

and

$$
\Omega_{p_{2}^{*}}=\left\{u \in E_{1}:\|u\|<p_{2}^{*}\right\}
$$

If $u \in P_{1} \cap \partial \Omega_{p_{2}^{*}}$, one has $\|u\|=p_{2}^{*}$ and

$$
\begin{aligned}
\|A u\| & \leq \frac{k_{0}}{k_{0}-k_{1}} \frac{m_{0}}{m_{0}-m_{1}} \frac{\lambda}{\Gamma(\alpha-1)} \int_{0}^{1}(1-\tau)^{\alpha-2} \varphi_{q}\left(\int_{0}^{\tau} f\left(s, u, u^{\prime}\right) d s\right) d \tau \\
& \leq \frac{k_{0}}{k_{0}-k_{1}} \frac{m_{0}}{m_{0}-m_{1}} \frac{\lambda}{\Gamma(\alpha-1)} \int_{0}^{1}(1-\tau)^{\alpha-2} \max \left\{C_{4}, \Lambda_{1}\left|u^{\prime}\right|\right\} \tau^{q-1} d \tau \\
& \leq \Lambda_{1} p_{2}^{*} \frac{k_{0}}{k_{0}-k_{1}} \frac{m_{0}}{m_{0}-m_{1}} \frac{\lambda \Gamma(q)}{\Gamma(\alpha+q-1)} \\
& =\|u\| .
\end{aligned}
$$

This implies our desired result.

Next, we deal with the existence of at least two distinct positive solutions to the fractional differential equation (2).

Theorem 5 Assume that all assumptions of Lemma 8 hold. Moreover, suppose that $f_{0}=\infty$ and $f_{\infty}=\infty$, and the condition (i) in Theorem 2 is satisfied. Then the fractional differential equation (2) has at least two distinct positive solutions $u_{1}, u_{2} \in P_{1}$.

Proof of Theorem 5 In view of $f_{0}=\infty$, there exists an $H_{1}$ such that $0<H_{1}<m$ and

$$
\begin{aligned}
f\left(t, u, u^{\prime}\right) & \geq \varphi_{p}\left(h\left(u+\left|u^{\prime}\right|\right)\right) \\
& \geq \varphi_{p}(h\|u\|) \quad \text { for }\left(t, u, u^{\prime}\right) \in[0,1] \times\left(0, \frac{k_{0}}{k_{0}-k_{1}} H_{1}\right] \times\left[-H_{1}, H_{1}\right],
\end{aligned}
$$

where $h$ is given by

$$
h \frac{m_{0}}{m_{0}-m_{1}} \frac{\lambda \Gamma(q)}{\Gamma(\alpha+q-1)} \geq 1 .
$$

Take

$$
\Omega_{H_{1}}=\left\{u \in E_{1}:\|u\|<H_{1}\right\} .
$$

If $u \in \Omega_{H_{1}}$ with $\|u\|=H_{1}$, it means that

$$
\sup _{t \in[0,1]}\left|u^{\prime}(t)\right| \leq\|u\|=H_{1} \quad \text { for } t \in[0,1]
$$


and

$$
\sup _{t \in[0,1]} u(t) \leq H_{1} \quad \text { for } t \in[0,1] .
$$

It follows from (24) and (25) that

$$
\begin{aligned}
\|A u\| & \geq\left|\max _{t \in[0,1]}(A u)^{\prime}(t)\right| \\
& =\frac{m_{0}}{m_{0}-m_{1}} \frac{\lambda}{\Gamma(\alpha-1)} \int_{0}^{1}(1-\tau)^{\alpha-2} \varphi_{q}\left(\int_{0}^{\tau} f\left(s, u, u^{\prime}\right) d s\right) d \tau \\
& \geq \frac{m_{0}}{m_{0}-m_{1}} \frac{\lambda}{\Gamma(\alpha-1)} \int_{0}^{1}(1-\tau)^{\alpha-2} \tau^{q-1} h\|u\| d \tau \\
& \geq h \frac{m_{0}}{m_{0}-m_{1}} \frac{\lambda \Gamma(q)}{\Gamma(\alpha+q-1)}\|u\|,
\end{aligned}
$$

which implies that

$$
\|A u\| \geq\|u\| \quad \text { for } u \in P_{1} \cap \partial \Omega_{H_{1}} .
$$

Let

$$
\Omega_{m}=\left\{u \in E_{1}:\|u\|<m\right\} .
$$

Then we obtain that (18) holds by using the condition (i) of Theorem 2. According to Lemma 2, the fractional differential equation (2) has a positive solution $u_{1}$ in $P_{1} \cap$ $\left(\bar{\Omega}_{m} \backslash \Omega_{H_{1}}\right)$.

It follows from $f_{\infty}=\infty$ that there exists an $H_{2}>\frac{4 k_{0}}{k_{0}-k_{1}} e$ such that

$$
f\left(t, u, u^{\prime}\right) \geq \varphi_{p}\left(k\left(u+\left|u^{\prime}\right|\right)\right) \geq \varphi_{p}(k\|u\|)
$$

where $t \in[0,1]$ and $u+\left|u^{\prime}\right| \geq H_{2}$. Moreover, $k$ satisfies that

$$
k \frac{m_{0}}{m_{0}-m_{1}} \frac{\lambda \Gamma(q)}{\Gamma(\alpha+q-1)} \geq 1
$$

Let

$$
\Omega_{H_{2}}=\left\{u \in \Omega_{H}: u+\left|u^{\prime}\right|<H_{2}\right\},
$$

then we see that $\bar{\Omega}_{m} \subset \Omega_{H_{2}}$.

For any $u \in P_{1} \cap \partial \Omega_{H_{2}}$, we have $u+\left|u^{\prime}\right|=H_{2}$. According to (26), we deduce that

$$
\begin{aligned}
\|A u\| & \geq \frac{m_{0}}{m_{0}-m_{1}} \frac{\lambda}{\Gamma(\alpha-1)} \int_{0}^{1}(1-\tau)^{\alpha-2} \varphi_{q}\left(\int_{0}^{\tau} f\left(s, u, u^{\prime}\right) d s\right) d \tau \\
& \geq \frac{m_{0}}{m_{0}-m_{1}} \frac{\lambda}{\Gamma(\alpha-1)} \int_{0}^{1}(1-\tau)^{\alpha-2} \tau^{q-1} k\|u\| d s
\end{aligned}
$$




$$
\begin{aligned}
& \geq k \frac{m_{0}}{m_{0}-m_{1}} \frac{\lambda \Gamma(q)}{\Gamma(\alpha+q-1)}\|u\| \\
& \geq\|u\| .
\end{aligned}
$$

Thus, it follows from (i) of Lemma 2 that the fractional differential equation (2) has at least a single positive solution $u_{2}$ in $P_{1} \cap\left(\bar{\Omega}_{H_{2}} \backslash \Omega_{m}\right)$ with

$$
m \leq\left\|u_{2}\right\| \quad \text { and } \quad u_{2}+\left|u_{2}^{\prime}\right| \leq H_{2} .
$$

It is easily seen that $u_{1}$ and $u_{2}$ are distinct.

By a closely similar way, we can obtain the following result.

Theorem 6 Assume that all assumptions of Lemma 8 hold. Moreover, suppose that $f^{0}=0$ and $f^{\infty}=0$, and the condition (ii) in Theorem 2 is satisfied, then the fractional differential equation (2) has at least two distinct positive solutions $u_{1}, u_{2} \in P_{1}$.

\section{Existence of triple or multiple solutions}

In this section, we will further discuss the existence of at least $3, n$ or $2 n-1$ positive solutions to $p$-Laplacian fractional differential equation (2) by using different fixed point theorems in cone.

For the notational convenience, we define

$$
\begin{aligned}
& M=\frac{m_{0}}{m_{0}-m_{1}} \frac{\lambda \Gamma(q)}{\Gamma(\alpha+q-1)}, \\
& N=\frac{m_{0}}{m_{0}-m_{1}} \frac{k_{1}}{k_{0}-k_{1}} \frac{\lambda \Gamma(q)}{\Gamma(\alpha+q-1)},
\end{aligned}
$$

and

$$
L=\frac{k_{0}}{k_{0}-k_{1}} \frac{m_{0}}{m_{0}-m_{1}} \frac{\lambda \Gamma(q)}{\Gamma(\alpha+q-1)} .
$$

\subsection{Existence of three solutions}

In this subsection, we investigate the existence of at least three distinct positive solutions of equation (2).

Theorem 7 Let $a, b$ and $c$ be constants such that $0<a<b<d \leq c$ and $b k_{0}<c k_{1}$. In addition, if all assumptions of Lemma 8 hold and $f\left(t, u_{1}, u_{2}\right)$ satisfies the following conditions:

(i) $f\left(t, u_{1}, u_{2}\right)<\varphi_{p}\left(\frac{a}{L}\right)$ for $\left(t, u_{1}, u_{2}\right) \in[0,1] \times[0, a] \times[-a, a]$;

(ii) $f\left(t, u_{1}, u_{2}\right)>\varphi_{p}\left(\frac{b}{N}\right)$ for $\left(t, u_{1}, u_{2}\right) \in[0,1] \times[b, d] \times[-c, c]$;

(iii) $f\left(t, u_{1}, u_{2}\right) \leq \varphi_{p}\left(\frac{c}{L}\right)$ for $\left(t, u_{1}, u_{2}\right) \in[0,1] \times[0, c] \times[-c, c]$.

Then the fractional differential equation (2)has at least triple positive solutions $u_{1}, u_{2}, u_{3} \in$ $P_{1}$ such that

$$
0<\left\|u_{1}\right\|<a, \quad b<\inf _{t \in[0,1]} u_{2}, \quad a<u_{3} \quad \text { with } \quad \inf _{t \in[0,1]} u_{3}<b .
$$


Proof of Theorem 7 By the virtue of the completely continuous operator $A$ and Lemma 3, we need to show that all conditions of Lemma 3 with respect to $A$ are satisfied.

Let

$$
q(u)=\inf _{t \in[0,1]} u(t) \quad \text { for } u \in \bar{P}_{1},
$$

then $q(u)$ is a nonnegative continuous concave function and satisfies

$$
q(u) \leq\|u\| \quad \text { for } u \in P_{1_{c}}=\left\{u \in P_{1}:\|u\| \leq c\right\} .
$$

Since

$$
u \in[0, c] \text { and } u^{\prime} \in[-c, c] \quad \text { for } u \in P_{1_{c}} \text {, }
$$

it follows from condition (iii) that

$$
\begin{aligned}
\|A u\| & \leq \frac{k_{0}}{k_{0}-k_{1}} \frac{m_{0}}{m_{0}-m_{1}} \frac{\lambda}{\Gamma(\alpha-1)} \int_{0}^{1}(1-\tau)^{\alpha-2} \varphi_{q}\left(\int_{0}^{\tau} f\left(s, u, u^{\prime}\right) d s\right) d \tau \\
& \leq \frac{c}{L} \frac{k_{0}}{k_{0}-k_{1}} \frac{m_{0}}{m_{0}-m_{1}} \frac{\lambda}{\Gamma(\alpha-1)} \int_{0}^{1}(1-\tau)^{\alpha-2} \tau d \tau \\
& \leq \frac{c}{L} \frac{k_{0}}{k_{0}-k_{1}} \frac{m_{0}}{m_{0}-m_{1}} \frac{\lambda \Gamma(q)}{\Gamma(\alpha+q-1)} \\
& \leq c
\end{aligned}
$$

which implies $A: \bar{P}_{1_{c}} \rightarrow \bar{P}_{1_{c}}$.

When $u \in P_{1_{a}}=\left\{u \in P_{1}:\|u\| \leq a\right\}$, it implies that $u \in[0, a]$ and $u^{\prime} \in[-a, a]$, from this, we can easily obtain that the conditions (ii) of Lemma 3 is true.

Let $d$ be a fixed constant such that $b<d \leq c$, then we have $q(d)=d>b$ and $\|d\|=d$. This means that

$$
d \in P_{1}(q, b, d)=\left\{u \in P_{1}: b \leq q(u),\|u\| \leq d\right\} .
$$

For any $u \in P_{1}(q, b, d)$, we get

$$
\|u\| \leq d \quad \text { and } \quad q(u)=\inf _{t \in[0,1]} u \geq b
$$

which implies that

$$
u \in[b, d] \text { and } u^{\prime} \in[-d, d] \text { for } t \in[0,1] .
$$

Hence, the condition (ii) gives that

$$
\begin{aligned}
q(A u) & =\inf _{t \in[0,1]} A u=\min _{t \in[0,1]}|(A u)(t)|=(A u)(0) \\
& =\frac{m_{0}}{m_{0}-m_{1}} \frac{\lambda}{\Gamma(\alpha-1)} \frac{k_{1}}{k_{0}-k_{1}} \int_{0}^{1}(1-\tau)^{\alpha-2} \varphi_{q}\left(\int_{0}^{\tau} f\left(s, u, u^{\prime}\right) d s\right) d \tau
\end{aligned}
$$




$$
\begin{aligned}
& =\frac{b}{N} \frac{m_{0}}{m_{0}-m_{1}} \frac{\lambda}{\Gamma(\alpha-1)} \frac{k_{1}}{k_{0}-k_{1}} \int_{0}^{1}(1-\tau)^{\alpha-2} \tau d \tau \\
& =\frac{b}{N} \frac{m_{0}}{m_{0}-m_{1}} \frac{\lambda \Gamma(q)}{\Gamma(\alpha+q-1)} \frac{k_{1}}{k_{0}-k_{1}} \\
& >b
\end{aligned}
$$

which means that the condition (i) of Lemma 3 holds.

For any $u \in P_{1}(q, b, c)$ with $\|A u\|>d$, it gives $\|u\| \leq c$ and $\inf _{t \in[0,1]} u \geq b$. By using the same argument as the above, we see that $q(A u)>b$. This implies that the condition (iii) of Lemma 3 is fulfilled.

Consequently, all conditions of Lemma 3 are verified. That is, the fractional differential equation (2) has at least three distinct solutions distributed as (27).

Corollary 1 Assume that all assumptions of Lemma 8 hold. If the condition (iii) in Theorem 7 is replaced by

$$
\text { (iii') } f^{\infty}=\lim _{\left(u_{1}, u_{2}\right) \rightarrow(\infty, \infty)} \sup _{t \in[0,1]} f\left(t, u_{1}, u_{2}\right) \leq \varphi_{p}\left(\frac{c}{L}\right) \quad \text { for } u_{1}, u_{2} \in P_{1} \text {, }
$$

then (27) in Theorem 7 also holds.

Proof of Corollary 1 We only need to prove that the condition (iii') implies the condition (iii) in Theorem 7. That is, assume that (iii') holds, then there exists a number $c^{*} \geq d^{*}$ such that

$$
f\left(t, u, u^{\prime}\right) \leq \varphi_{p}\left(\frac{c^{*}}{L}\right) \quad \text { for }\left(t, u, u^{\prime}\right) \in[0,1] \times\left[0, \frac{k_{0}}{k_{0}-k_{1}} c^{*}\right] \times\left[-c^{*}, c^{*}\right]
$$

Conversely, we suppose that for any $c^{*} \geq d^{*}$, there exists

$$
\left(u_{c}, u_{c}^{\prime}\right) \in\left[0, \frac{k_{0}}{k_{0}-k_{1}} c^{*}\right] \times\left[-c^{*}, c^{*}\right]
$$

such that

$$
f\left(t, u_{c}, u_{c}^{\prime}\right)>\varphi_{p}\left(\frac{c^{*}}{L}\right) \text { for } t \in[0,1]
$$

Take

$$
c_{n}^{*}>d^{*} \quad(n=1,2, \ldots) \text { with } c_{n}^{*} \rightarrow \infty .
$$

Then there exists

$$
\left(u_{n}, u_{n}^{\prime}\right) \in\left[0, \frac{k_{0}}{k_{0}-k_{1}} c_{n}^{*}\right] \times\left[-c_{n}^{*}, c_{n}^{*}\right]
$$

such that

$$
f\left(t, u_{n}, u_{n}^{\prime}\right)>\varphi_{p}\left(\frac{c_{n}^{*}}{L}\right) \quad \text { for } t \in[0,1]
$$


and

$$
\lim _{n \rightarrow \infty} f\left(t, u_{n}, u_{n}^{\prime}\right)=\infty \quad \text { for } t \in[0,1]
$$

Since the condition (iii') holds, there is a $\tau>0$ such that

$$
\begin{aligned}
& f\left(t, u, u^{\prime}\right) \leq \varphi_{p}\left(\frac{\left|u^{\prime}\right|}{L}\right) \\
& \quad \text { for }\left(t, u, u^{\prime}\right) \in[0,1] \times\left[\frac{k_{0}}{k_{0}-k_{1}} \tau, \infty\right) \times(-\infty, \tau] \cup[\tau, \infty) .
\end{aligned}
$$

Thus, we have

$$
\left|u_{n}^{\prime}(t)\right| \leq \tau \quad \text { for } t \in[0,1]
$$

and

$$
u_{n}(t) \leq \frac{k_{0}}{k_{0}-k_{1}} \tau \quad \text { for } t \in[0,1]
$$

Otherwise, if

$$
\left|u_{n}^{\prime}(t)\right|>\tau \quad \text { and } \quad u_{n}(t)>\frac{k_{0}}{k_{0}-k_{1}} \tau \quad \text { for } t \in[0,1] \text {, }
$$

it follows from (31) that

$$
f\left(t, u_{n}, u_{n}^{\prime}\right) \leq \varphi_{p}\left(\frac{\left|u_{n}^{\prime}\right|}{L}\right) \leq \varphi_{p}\left(\frac{c_{n}^{*}}{L}\right) \quad \text { for } t \in[0,1]
$$

which contradicts inequality (29).

Let

$$
W=\max _{\left(t, u, u^{\prime}\right) \in[0,1] \times\left[0, \frac{k_{0}}{k_{0}-k_{1}} \tau\right] \times[-\tau, \tau]} f\left(t, u, u^{\prime}\right),
$$

so we have

$$
f\left(t, u_{n}, u_{n}^{\prime}\right) \leq W \quad(n=1,2, \ldots) .
$$

This apparently contradicts formula (30). Consequently, we complete the proof.

\subsection{Existence of arbitrary $\boldsymbol{n}$ solutions}

In this subsection, the existence criteria for at least three or arbitrary $n$ positive solutions to $p$-Laplacian fractional differential equation (2) are obtained.

We define the nonnegative, increasing, continuous functionals $\gamma_{1}, \beta_{1}$ and $\alpha_{1}$ by

$$
\gamma_{1}(u)=\beta_{1}(u)=\max \left\{\inf _{t \in[0,1]} u, \inf _{t \in[0,1]}\left|u^{\prime}\right|\right\} \quad \text { for } u \in P_{1}
$$


and

$$
\alpha_{1}(u)=\max \left\{\sup _{t \in[0,1]} u, \sup _{t \in[0,1]}\left|u^{\prime}\right|\right\} \quad \text { for } u \in P_{1} \text {, }
$$

so

$\gamma_{1}(u)=\beta_{1}(u) \leq \alpha_{1}(u) \quad$ for each $u \in P_{1}$.

Since

$$
\begin{aligned}
\inf _{t \in[0,1]} u(t) & =\min _{t \in[0,1]} u(t)=u(0) \\
& =\frac{m_{0}}{m_{0}-m_{1}} \frac{\lambda}{\Gamma(\alpha-1)} \frac{k_{1}}{k_{0}-k_{1}} \int_{0}^{1}(1-\tau)^{\alpha-2} \int_{0}^{\tau} h(s) d s d \tau \\
& \geq \frac{k_{1}}{k_{0}} \frac{m_{0}}{m_{0}-m_{1}} \frac{\lambda}{\Gamma(\alpha-1)} \frac{k_{0}}{k_{0}-k_{1}} \int_{0}^{1}(1-\tau)^{\alpha-2} \int_{0}^{\tau} h(s) d s d \tau \\
& =\frac{k_{1}}{k_{0}} \max _{t \in[0,1]} u(t)=\|u\|_{1},
\end{aligned}
$$

and

$$
\begin{aligned}
\inf _{t \in[0,1]}\left|u^{\prime}(t)\right| & =u^{\prime}(0) \\
& =\frac{m_{1}}{m_{0}-m_{1}} \frac{\lambda}{\Gamma(\alpha-1)} \int_{0}^{1}(1-\tau)^{\alpha-2} \int_{0}^{\tau} h(s) d s d \tau \\
& =\frac{m_{1}}{m_{0}} \frac{m_{0}}{m_{0}-m_{1}} \frac{\lambda}{\Gamma(\alpha-1)} \int_{0}^{1}(1-\tau)^{\alpha-2} \int_{0}^{\tau} h(s) d s d \tau \\
& =\frac{m_{1}}{m_{0}}\|u\|_{2} .
\end{aligned}
$$

Hence, we have

$$
\|u\| \leq \max \left\{\frac{m_{0}}{m_{1}}, \frac{k_{0}}{k_{1}}\right\} \gamma_{1}(u)=\gamma \gamma_{1}(u) \quad \text { for all } u \in P_{1},
$$

where $\gamma=\max \left\{\frac{m_{0}}{m_{1}}, \frac{k_{0}}{k_{1}}\right\}$.

Theorem 8 Assume that there exist real numbers $a^{\prime}, b^{\prime}, c^{\prime}$ with $a^{\prime}<b^{\prime}<c^{\prime}$ such that $0<$ $k_{0} b^{\prime}<a^{\prime} k_{1}$. In addition, if all assumptions of Lemma 8 hold and $f\left(t, u_{1}, u_{2}\right)$ satisfies the following conditions:

(i) $f\left(t, u_{1}, u_{2}\right)<\varphi_{p}\left(\frac{c^{\prime}}{L}\right)$ for $\left(t, u_{1}, u_{2}\right) \in[0,1] \times\left[0, \gamma c^{\prime}\right] \times\left[-\gamma c^{\prime}, \gamma c^{\prime}\right]$;

(ii) $f\left(t, u_{1}, u_{2}\right)>\varphi_{p}\left(\frac{b^{\prime}}{N}\right)$ for $\left(t, u_{1}, u_{2}\right) \in[0,1] \times\left[b^{\prime}, \gamma b^{\prime}\right] \times\left[-\gamma b^{\prime}, \gamma b^{\prime}\right]$;

(iii) $f\left(t, u_{1}, u_{2}\right)<\varphi_{p}\left(\frac{a^{\prime}}{L}\right)$ for $\left(t, u_{1}, u_{2}\right) \in[0,1] \times\left[0, a^{\prime}\right] \times\left[-a^{\prime}, a^{\prime}\right]$.

Then the fractional differential equation (2) has at least three distinct positive solutions $u_{1}, u_{2}, u_{3} \in \bar{P}_{1}\left(\gamma_{1}, c^{\prime}\right)$ such that

$$
\begin{aligned}
& 0<\left\|u_{1}\right\|<a^{\prime}<\left\|u_{2}\right\|, \\
& \max \left\{\inf _{t \in[0,1]} u_{2}, \inf _{t \in[0,1]}\left|u_{2}^{\prime}\right|\right\}<b^{\prime}<\max \left\{\inf _{t \in[0,1]} u_{3}, \inf _{t \in[0,1]}\left|u_{3}^{\prime}\right|\right\}<c^{\prime} .
\end{aligned}
$$


Proof of Theorem 8 We only need to check all conditions of Lemma 4 are fulfilled with respect to the operator $A$. By using a similar way as to the proof of (28), we can obtain that

$$
A: \bar{P}_{1}\left(\gamma_{1}, c^{\prime}\right) \rightarrow \bar{P}_{1} .
$$

For arbitrary $u \in \partial P_{1}\left(\gamma_{1}, c^{\prime}\right)$, one has

$$
\gamma_{1}(u)=\max \left\{\inf _{t \in[0,1]} u, \inf _{t \in[0,1]}\left|u^{\prime}\right|\right\}=c^{\prime}
$$

and

$$
\|u\| \leq \gamma \gamma_{1}(u)=\gamma c^{\prime} .
$$

This implies that

$$
0 \leq u \leq \gamma c^{\prime}, \quad t \in[0,1],
$$

and

$$
-\gamma c^{\prime} \leq u^{\prime} \leq \gamma c^{\prime}, \quad t \in[0,1] .
$$

According to the condition (i), it gives

$$
\begin{aligned}
\gamma_{1}(A u) & =\max \left\{\inf _{t \in[0,1]} A u, \inf _{t \in[0,1]}\left|A u^{\prime}\right|\right\} \\
& \leq \frac{m_{0}}{m_{0}-m_{1}} \frac{\lambda}{\Gamma(\alpha-1)} \frac{k_{0}}{k_{0}-k_{1}} \int_{0}^{1}(1-\tau)^{\alpha-2} \varphi_{q}\left(\int_{0}^{\tau} f\left(s, u, u^{\prime}\right) d s\right) d \tau \\
& \leq \frac{c^{\prime}}{L} \frac{m_{0}}{m_{0}-m_{1}} \frac{\lambda}{\Gamma(\alpha-1)} \frac{k_{0}}{k_{0}-k_{1}} \int_{0}^{1}(1-\tau)^{\alpha-2} \tau d \tau \\
& =\frac{c^{\prime}}{L} \frac{m_{0}}{m_{0}-m_{1}} \frac{\lambda \Gamma(q)}{\Gamma(\alpha+q-1)} \frac{k_{0}}{k_{0}-k_{1}} \\
& <c^{\prime} .
\end{aligned}
$$

We see that $\gamma_{1}(A u)<c^{\prime}$ for $u \in \partial P_{1}\left(\gamma_{1}, c^{\prime}\right)$.

For any $u \in \partial P_{1}\left(\beta_{1}, b^{\prime}\right)$, it gives

$$
\beta_{1}(u)=\max \left\{\inf _{t \in[0,1]} u, \inf _{t \in[0,1]}\left|u^{\prime}\right|\right\}=b^{\prime},
$$

and

$$
\|u\| \leq \gamma \beta_{1}(u)=\gamma \gamma_{1}(u)=\gamma b^{\prime} .
$$

This implies that

$$
b^{\prime} \leq u \leq \gamma b^{\prime}, \quad t \in[0,1],
$$


and

$$
-\gamma b^{\prime} \leq u^{\prime} \leq \gamma b^{\prime}, \quad t \in[0,1]
$$

Making use of the condition (ii), we get

$$
\begin{aligned}
\beta_{1}(A u)= & \max \left\{\inf _{t \in[0,1]} A u, \inf _{t \in[0,1]}\left|A u^{\prime}\right|\right\} \\
= & \max \left\{\frac{k_{1}}{k_{0}-k_{1}} \frac{m_{0}}{m_{0}-m_{1}}, \frac{m_{1}}{m_{0}-m_{1}}\right\} \frac{\lambda}{\Gamma(\alpha-1)} \\
& \times \int_{0}^{1}(1-\tau)^{\alpha-2} \varphi_{q}\left(\int_{0}^{\tau} f\left(s, u, u^{\prime}\right) d s\right) d \tau \\
\geq & \frac{b^{\prime}}{N} \frac{k_{1}}{k_{0}-k_{1}} \frac{m_{0}}{m_{0}-m_{1}} \frac{\lambda}{\Gamma(\alpha-1)} \int_{0}^{1}(1-\tau)^{\alpha-2} \tau d \tau \\
= & \frac{b^{\prime}}{N} \frac{k_{1}}{k_{0}-k_{1}} \frac{m_{0}}{m_{0}-m_{1}} \frac{\lambda \Gamma(q)}{\Gamma(\alpha+q-1)} \\
= & b^{\prime} .
\end{aligned}
$$

So we have $\beta_{1}(A u)>b^{\prime}$ for $u \in \partial P_{1}\left(\beta_{1}, b^{\prime}\right)$.

We now show that $P_{1}\left(\alpha_{1}, a^{\prime}\right) \neq \varnothing$ and $\alpha_{1}(A u)<a^{\prime}$ for arbitrary $u \in \partial P_{1}\left(\alpha_{1}, a^{\prime}\right)$. Since $\frac{a^{\prime}}{2} \in$ $P_{1}\left(\alpha_{1}, a^{\prime}\right)$, for $u \in \partial P_{1}\left(\alpha_{1}, a^{\prime}\right)$, we have

$$
\alpha_{1}(u)=\max \left\{\sup _{t \in[0,1]} u, \sup _{t \in[0,1]}\left|u^{\prime}\right|\right\}=a^{\prime}
$$

which gives

$$
0 \leq u \leq a^{\prime} \quad \text { and } \quad-a^{\prime} \leq u^{\prime} \leq a^{\prime} \quad \text { for } t \in[0,1]
$$

It follows from the assumption (iii) that

$$
\begin{aligned}
\alpha_{1}(A u) & \leq \frac{k_{0}}{k_{0}-k_{1}} \frac{m_{0}}{m_{0}-m_{1}} \frac{\lambda}{\Gamma(\alpha-1)} \int_{0}^{1}(1-\tau)^{\alpha-2} \varphi_{q}\left(\int_{0}^{\tau} f\left(s, u, u^{\prime}\right) d s\right) d \tau \\
& \leq \frac{a^{\prime}}{L} \frac{k_{0}}{k_{0}-k_{1}} \frac{m_{0}}{m_{0}-m_{1}} \frac{\lambda}{\Gamma(\alpha-1)} \int_{0}^{1}(1-\tau)^{\alpha-2} \tau d \tau \\
& =a^{\prime} .
\end{aligned}
$$

All conditions in Lemma 4 are satisfied. From (S1) and (S2), we know that solutions of equation (2) do not vanish identically on any closed subinterval of $[0,1]$. Consequently, equation (2) has at least three distinct positive solutions $u_{1}, u_{2}$, and $u_{3}$ belonging to $\bar{P}\left(\gamma_{1}, c^{\prime}\right)$ distributed as (32).

The following result is regarded as a corollary of Theorem 8.

Corollary 2 Assume that all assumptions of Lemma 8 hold and $f$ satisfies the following conditions:

(i) $f^{0}=0$ and $f^{\infty}=0$; 
(ii) there exists a $c_{0}>0$ such that

$$
f\left(s, u_{1}, u_{2}\right)>\varphi_{p}\left(\frac{1}{\gamma} \frac{c_{0}}{N}\right) \quad \text { for }\left(t, u_{1}, u_{2}\right) \in[0,1] \times\left[\frac{c_{0}}{\gamma}, c_{0}\right] \times\left[-c_{0}, c_{0}\right] .
$$

Then the fractional differential equation (2) has at least three distinct positive solutions.

Proof of Corollary 2 Let $b^{\prime}=\frac{1}{\gamma} c_{0}$. It follows from the condition (ii) that

$$
f\left(t, u_{1}, u_{2}\right)>\varphi_{p}\left(\frac{b^{\prime}}{N}\right) \quad \text { for }\left(t, u_{1}, u_{2}\right) \in[0,1] \times\left[b^{\prime}, b^{\prime} \gamma\right] \times\left[-\gamma b^{\prime}, \gamma b^{\prime}\right],
$$

which implies that the condition (ii) of Theorem 8 holds.

We choose a sufficiently small $\varepsilon_{5}>0$ such that

$$
\varepsilon_{5} L=\varepsilon_{5} \frac{k_{0}}{k_{0}-k_{1}} \frac{m_{0}}{m_{0}-m_{1}} \frac{\lambda \Gamma(q)}{\Gamma(\alpha+q-1)}<1 .
$$

In view of $f^{0}=0$, there exists a sufficiently small $k_{1}^{*}>0$ such that

$$
f\left(t, u_{1}, u_{2}\right) \leq \varphi_{p}\left(\varepsilon_{5}\left|u_{2}\right|\right) \quad \text { for }\left(t, u_{1}, u_{2}\right) \in[0,1] \times\left[0, \frac{k_{0}}{k_{0}-k_{1}} k_{1}^{*}\right] \times\left[-k_{1}^{*}, k_{1}^{*}\right] .
$$

Without loss of generality, let $k_{1}^{*}=a^{\prime}<b^{\prime}$. Because of $\max _{t \in[0,1]}\left|u_{2}\right| \leq a^{\prime}$, we have $\max _{t \in[0,1]} u_{1} \leq \frac{k_{0}}{k_{0}-k_{1}} \max _{t \in[0,1]}\left|u_{2}\right| \leq \frac{k_{0}}{k_{0}-k_{1}} a^{\prime}$. Thus, it follows from (33) and (34) that

$$
\begin{aligned}
& f\left(t, u_{1}, u_{2}\right) \leq \varphi_{p}\left(\varepsilon_{5}\left|u_{2}\right|\right) \leq \varphi_{p}\left(\varepsilon_{5} a^{\prime}\right)<\varphi_{p}\left(\frac{a^{\prime}}{L}\right) \\
& \quad \text { for }\left(t, u_{1}, u_{2}\right) \in[0,1] \times\left[0, \frac{k_{0}}{k_{0}-k_{1}} a^{\prime}\right] \times\left[-a^{\prime}, a^{\prime}\right],
\end{aligned}
$$

which implies that the condition (iii) of Theorem 8 holds.

Choose $\varepsilon_{6}$ sufficiently small such that

$$
\varepsilon_{6} \gamma L=\varepsilon_{6} \gamma \frac{k_{0}}{k_{0}-k_{1}} \frac{m_{0}}{m_{0}-m_{1}} \frac{\lambda \Gamma(q)}{\Gamma(\alpha+q-1)}<1
$$

By using the continuity of $f$, there exists a constant $C^{*}$ such that

$$
f\left(t, u_{1}, u_{2}\right) \leq \varphi_{p}\left(C^{*}\right) \quad \text { for }\left(t, u_{1}, u_{2}\right) \in[0,1] \times\left[0, \frac{k_{0}}{k_{0}-k_{1}} \gamma c^{\prime}\right] \times\left[-\gamma c^{\prime}, \gamma c^{\prime}\right] .
$$

Since $f^{\infty}=0$, there exists a $k_{2}>L C^{*}$ sufficiently large such that

$$
\begin{aligned}
& f\left(t, u_{1}, u_{2}\right) \leq \varphi_{p}\left(\varepsilon_{6}\left|u_{2}\right|\right) \\
& \quad \text { for }\left(t, u_{1}, u_{2}\right) \in[0,1] \times\left[\frac{k_{0}}{k_{0}-k_{1}} k_{2},+\infty\right) \times\left[k_{2},+\infty\right) \cup\left(-\infty, k_{2}\right] .
\end{aligned}
$$

Without loss of generality, let $k_{2}>\frac{b^{\prime}}{\gamma}$ and $c^{\prime}=k_{2}$, so we see if

$$
\left(t, u_{1}, u_{2}\right) \in[0,1] \times\left[0, \frac{k_{0}}{k_{0}-k_{1}} \gamma c^{\prime}\right] \times\left[-\gamma c^{\prime},-c^{\prime}\right] \cup\left[c^{\prime}, \gamma c^{\prime}\right],
$$


then

$$
f\left(t, u_{1}, u_{2}\right) \leq \varphi_{p}\left(\varepsilon_{6}\left|u_{2}\right|\right) \leq \varphi_{p}\left(\varepsilon_{6} \gamma c^{\prime}\right)<\varphi_{p}\left(\frac{c^{\prime}}{L}\right)
$$

Moreover, in view of (35), one has

$$
\begin{aligned}
f\left(t, u_{1}, u_{2}\right) & \leq \varphi_{p}\left(C^{*}\right)<\varphi_{p}\left(\frac{k_{2}}{L}\right) \\
& =\varphi_{p}\left(\frac{c^{\prime}}{L}\right) \quad \text { for }\left(t, u_{1}, u_{2}\right) \in[0,1] \times\left[0, \frac{k_{0}}{k_{0}-k_{1}} \gamma c^{\prime}\right] \times\left[-\gamma c^{\prime}, \gamma c^{\prime}\right] .
\end{aligned}
$$

From (36) and (37), we see that the condition (i) of Theorem 8 is fulfilled. Hence, equation (2) has at least three distinct positive solutions according to Theorem 8.

According to Theorem 8, we can prove that the existence for multiple positive solutions to the equation (2) when conditions (i), (ii) and (iii) are modified appropriately on $f$.

Theorem 9 If there exist constant numbers $a_{i}^{\prime}$, $b_{i}^{\prime}$ and $c_{i}^{\prime}$ such that $0<a_{1}^{\prime}<b_{1}^{\prime}<c_{1}^{\prime}<\cdots<$ $a_{n}^{\prime}<b_{n}^{\prime}<c_{n}^{\prime}$ together with

$$
0<k_{0} b_{1}^{\prime}<c_{1}^{\prime} k_{1}<k_{0} b_{2}^{\prime}<c_{2}^{\prime} k_{1}<\cdots<k_{0} b_{n}^{\prime}<c_{n}^{\prime} k_{1}, \quad n \in \mathbb{N}
$$

where $i=1,2, \ldots, n$. In addition, if all assumptions of Lemma 8 hold and the function $f$ satisfies:

(i) $f\left(t, u_{1}, u_{2}\right)<\varphi_{p}\left(\frac{c_{i}^{\prime}}{L}\right)$ for $\left(t, u_{1}, u_{2}\right) \in[0,1] \times\left[0, \gamma c_{i}^{\prime}\right] \times\left[-\gamma c_{i}^{\prime}, \gamma c_{i}^{\prime}\right]$;

(ii) $f\left(t, u_{1}, u_{2}\right)>\varphi_{p}\left(\frac{b_{i}^{\prime}}{N}\right)$ for $\left(t, u_{1}, u_{2}\right) \in[0,1] \times\left[b_{i}^{\prime}, \gamma b_{i}^{\prime}\right] \times\left[-\gamma b_{i}^{\prime}, \gamma b_{i}^{\prime}\right]$;

(iii) $f\left(t, u_{1}, u_{2}\right)<\varphi_{p}\left(\frac{a_{i}^{\prime}}{L}\right)$ for $\left(t, u_{1}, u_{2}\right) \in[0,1] \times\left[0, a_{i}^{\prime}\right] \times\left[-a_{i}^{\prime}, a_{i}^{\prime}\right]$.

Then the fractional differential equation (2) has at least $n$ distinct positive solutions.

Proof of Theorem 9 By using almost same technique as to the proof of Theorem 9 in [15].

By virtue of Lemma 4, we can obtain the following results by using the similar way as to those of Theorem 8 .

Theorem 10 Assume that there exist positive numbers $a^{\prime}, b^{\prime}, c^{\prime}$ with $a^{\prime}<b^{\prime}<c^{\prime}$ such that $c^{\prime} k_{1}<b^{\prime} k_{0}$. In addition, if all assumptions of Lemma 8 hold and $f\left(t, u_{1}, u_{2}\right)$ satisfies the following conditions:

(i) $f\left(t, u_{1}, u_{2}\right)>\varphi_{p}\left(\frac{c^{\prime}}{N}\right)$ for $\left(t, u_{1}, u_{2}\right) \in[0,1] \times\left[0, \gamma c^{\prime}\right] \times\left[-\gamma c^{\prime}, \gamma c^{\prime}\right]$;

(ii) $f\left(t, u_{1}, u_{2}\right)<\varphi_{p}\left(\frac{b^{\prime}}{L}\right)$ for $\left(t, u_{1}, u_{2}\right) \in[0,1] \times\left[b^{\prime}, \gamma b^{\prime}\right] \times\left[-\gamma b^{\prime}, \gamma b^{\prime}\right]$;

(iii) $f\left(t, u_{1}, u_{2}\right)>\varphi_{p}\left(\frac{a^{\prime}}{N}\right)$ for $\left(t, u_{1}, u_{2}\right) \in[0,1] \times\left[0, a^{\prime}\right] \times\left[-a^{\prime}, a^{\prime}\right]$.

Then the fractional differential equation (2) has at least three distinct positive solutions $u_{1}, u_{2}, u_{3} \in \bar{P}_{1}\left(\gamma_{1}, c^{\prime}\right)$ such that

$$
\begin{aligned}
& 0 \leq\left\|u_{1}\right\|<a^{\prime}<\left\|u_{2}\right\|, \\
& \max \left\{\inf _{t \in[0,1]} u_{2}, \inf _{t \in[0,1]}\left|u_{2}^{\prime}\right|\right\}<b^{\prime}<\max \left\{\inf _{t \in[0,1]} u_{3}, \inf _{t \in[0,1]}\left|u_{3}^{\prime}\right|\right\}<c^{\prime} .
\end{aligned}
$$


It also follows from Theorem 10 that we can obtain the following corollary and theorem, respectively.

Corollary 3 Assume that all assumptions of Lemma 8 hold and $f$ satisfies conditions:

(i) $f_{0}=\infty, f_{\infty}=\infty$;

(ii) there exists $c_{0}>0$ such that $f\left(t, u_{1}, u_{2}\right)<\varphi_{p}\left(\frac{1}{\gamma} \frac{c_{0}}{M}\right)$ for $\left(t, u_{1}, u_{2}\right) \in[0,1] \times\left[\frac{c_{0}}{\lambda}, c_{0}\right] \times\left[-c_{0}, c_{0}\right]$.

Then the fractional differential equation (2) has at least three distinct positive solutions.

Theorem 11 Assume that all assumptions of Lemma 8 hold and there are positive numbers $a_{i}^{\prime}, b_{i}^{\prime}, c_{i}^{\prime}$ such that $a_{1}^{\prime}<b_{1}^{\prime}<c_{1}^{\prime}<\cdots<a_{n}^{\prime}<b_{n}^{\prime}<c_{n}^{\prime}$ together with

$$
0<k_{0} b_{1}^{\prime}<c_{1}^{\prime} k_{1}<k_{0} b_{2}^{\prime}<c_{2}^{\prime} k_{1}<\cdots<k_{0} b_{n}^{\prime}<c_{n}^{\prime} k_{1}, \quad n \in \mathbb{N},
$$

where $i=1,2, \ldots, n$. In addition, $f\left(t, u_{1}, u_{2}\right)$ satisfies the following conditions:

(i) $f\left(t, u_{1}, u_{2}\right)>\varphi_{p}\left(\frac{c_{i}^{\prime}}{N}\right)$ for $\left(t, u_{1}, u_{2}\right) \in[0,1] \times\left[0, \gamma c_{i}^{\prime}\right] \times\left[-\gamma c_{i}^{\prime}, \gamma c_{i}^{\prime}\right]$;

(ii) $f\left(t, u_{1}, u_{2}\right)<\varphi_{p}\left(\frac{b_{i}^{\prime}}{L}\right)$ for $\left(t, u_{1}, u_{2}\right) \in[0,1] \times\left[b_{i}^{\prime}, \gamma b_{i}^{\prime}\right] \times\left[-\gamma b_{i}^{\prime}, \gamma b_{i}^{\prime}\right]$;

(iii) $f\left(t, u_{1}, u_{2}\right)>\varphi_{p}\left(\frac{a_{i}^{\prime}}{N}\right)$ for $\left(t, u_{1}, u_{2}\right) \in[0,1] \times\left[0, a_{i}^{\prime}\right] \times\left[-a_{i}^{\prime}, a_{i}^{\prime}\right]$.

Then the fractional differential equation (2) has at least $n$ distinct positive solutions.

\subsection{Existence of arbitrary $2 n-1$ solutions}

In this subsection, the existence of at least three or arbitrary odd positive solutions to $p$-Laplacian differential equation (2) are established.

Define the nonnegative continuous convex functionals $\phi$ and $\beta$, concave functional $\lambda$ and functional $\varphi$ on $P_{1}$ by

$$
\begin{aligned}
& \phi(u)=\max \left\{\sup _{t \in[0,1]} u, \sup _{t \in[0,1]}\left|u^{\prime}\right|\right\}, \\
& \beta(u)=\varphi(u)=\sup _{t \in[0,1]} u,
\end{aligned}
$$

and

$$
\lambda(u)=\inf _{t \in[0,1]}\left|u^{\prime}\right| .
$$

Theorem 12 Assume that all assumptions of Lemma 8 hold and there exist constants $a^{*}$, $b^{*}, d^{*}$ such that $0<a^{*}<b^{*}<\frac{k_{1}}{k_{0}} d^{*}$. In addition, $f\left(t, u_{1}, u_{2}\right)$ satisfies the following conditions:

(i) $f\left(t, u_{1}, u_{2}\right) \leq \varphi_{p}\left(\frac{d^{*}}{L}\right)$ for all $\left(t, u_{1}, u_{2}\right) \in[0,1] \times\left[0, d^{*}\right] \times\left[-d^{*}, d^{*}\right]$;

(ii) $f\left(t, u_{1}, u_{2}\right)>\varphi_{p}\left(\frac{b^{*}}{N}\right)$ for all $\left(t, u_{1}, u_{2}\right) \in[0,1] \times\left[b^{*}, d^{*}\right] \times\left[-d^{*}, d^{*}\right]$;

(iii) $f\left(t, u_{1}, u_{2}\right)<\varphi_{p}\left(\frac{a^{*}}{M}\right)$ for all $\left(t, u_{1}, u_{2}\right) \in[0,1] \times\left[0, a^{*}\right] \times\left[-d^{*}, d^{*}\right]$.

Then the fractional differential equation (2) has at least three distinct positive solutions $u_{1}$, $u_{2}, u_{3}$ such that

$$
\begin{aligned}
& \left\|x_{i}\right\| \leq d^{*} \quad \text { for } i=1,2,3, \quad b^{*}<\inf _{t \in[0,1]} u_{1}, \quad a^{*}<\sup _{t \in[0,1]} u_{2}, \\
& \inf _{t \in[0,1]} u_{2}<b^{*} \quad \text { with } \quad \sup _{t \in[0,1]} u_{3}<a^{*} .
\end{aligned}
$$


Proof of Theorem 12 It suffices to show that all conditions of Lemma 5 hold with respect to the completely continuous operator $A$.

For arbitrary $u \in P_{1}$, we have $\lambda(u) \leq \varphi(u)$ and $\|u\|=\phi(u)$. This implies that the inequality (3) of Lemma 5 is satisfied.

In the following, we show that $A: \overline{P_{1}\left(\phi, d^{*}\right)} \rightarrow \overline{P_{1}\left(\phi, d^{*}\right)}$.

For any $u \in \overline{P_{1}\left(\phi, d^{*}\right)}$, from $\phi(u)=\|u\| \leq d^{*}$ and the assumption (i), we have

$$
\begin{aligned}
\|A u\| & \leq \frac{k_{0}}{k_{0}-k_{1}} \frac{m_{0}}{m_{0}-m_{1}} \frac{\lambda}{\Gamma(\alpha-1)} \int_{0}^{1}(1-\tau)^{\alpha-2} \varphi_{q}\left(\int_{0}^{\tau} f\left(s, u, u^{\prime}\right) d s\right) d \tau \\
& =\frac{d^{*}}{L} \frac{k_{0}}{k_{0}-k_{1}} \frac{m_{0}}{m_{0}-m_{1}} \frac{\lambda \Gamma(q)}{\Gamma(\alpha+q-1)} \\
& =d^{*} .
\end{aligned}
$$

It remains to show that assumptions (i)-(iii) of Lemma 5 are fulfilled with respect to the operator $A$.

Let $u \equiv k b^{*}$, where $k=\frac{k_{0}}{k_{1}}$. It is obvious that $k>1, u=k b^{*}>b^{*}$ and $\beta(u)=k b^{*}$. We see that $b^{*}<\frac{k_{1}}{k_{0}} d^{*}$ that $\phi(u)=k b^{*}<d^{*}$. So we have

$$
\left\{u \in P_{1}\left(\phi, \beta, \lambda, b^{*}, k b^{*}, d^{*}\right): \lambda(x)>b^{*}\right\} \neq \emptyset .
$$

For any $u \in P_{1}\left(\phi, \beta, \lambda, b^{*}, k b^{*}, d^{*}\right)$, we get $b^{*} \leq u \leq d^{*}$ and $-d^{*} \leq u^{\prime} \leq d^{*}$ for all $t \in[0,1]$. It follows from the assumption (ii) that

$$
\begin{aligned}
\lambda(A u) & =(A u)^{\prime}(0) \\
& \geq \frac{b^{*}}{N} \frac{k_{1}}{k_{0}-k_{1}} \frac{m_{0}}{m_{0}-m_{1}} \frac{\lambda}{\Gamma(\alpha-1)} \int_{0}^{1}(1-\tau)^{\alpha-2} \tau d \tau \\
& =\frac{b^{*}}{N} \frac{k_{1}}{k_{0}-k_{1}} \frac{m_{0}}{m_{0}-m_{1}} \frac{\lambda \Gamma(q)}{\Gamma(\alpha+q-1)} \\
& =b^{*},
\end{aligned}
$$

which implies that assumption (i) of Lemma 5 is satisfied.

For any $u \in P_{1}\left(\phi, \lambda, b^{*}, d^{*}\right)$ with $\beta(A u)>k b^{*}$, we have $b^{*} \leq u \leq d^{*}$ and $-d^{*} \leq u^{\prime} \leq d^{*}$ for $t \in[0,1]$. So we have

$$
\lambda(A u)>b^{*} .
$$

This implies that assumption (ii) of Lemma 5 is fulfilled.

Since $\varphi(0)=0<a^{*}$, we have $0 \notin R\left(\phi, \varphi, a^{*}, d^{*}\right)$. If

$$
u \in R\left(\phi, \varphi, a^{*}, d^{*}\right) \quad \text { with } \quad \varphi(u)=\sup _{t \in[0,1]} u=a^{*}
$$

it reduces to

$$
0 \leq u \leq a^{*} \quad \text { and } \quad-d^{*} \leq u^{\prime} \leq d^{*} \quad \text { for all } t \in[0,1] .
$$


Hence, we have

$$
\begin{aligned}
\lambda(A u) & =\frac{m_{0}}{m_{0}-m_{1}} \frac{\lambda}{\Gamma(\alpha-1)} \int_{0}^{1}(1-\tau)^{\alpha-2} \varphi_{q}\left(\int_{0}^{\tau} f\left(s, u, u^{\prime}\right) d s\right) d \tau \\
& \leq \frac{a^{*}}{M} \frac{m_{0}}{m_{0}-m_{1}} \frac{\lambda}{\Gamma(\alpha-1)} \int_{0}^{1}(1-\tau)^{\alpha-2} \tau d \tau \\
& =a^{*} .
\end{aligned}
$$

All assumptions of Lemma 5 are satisfied. Consequently, we complete the proof.

Corollary 4 Assume that all assumptions of Lemma 8 hold and the condition (i) in Theorem 12 is replaced by (i'), then the conclusion of Theorem 12 also holds.

Similar to the proof of Theorem 9 by mathematical induction, we have the following.

Theorem 13 Assume that all assumptions of Lemma 8 hold and there exist constants $a_{i}^{*}$, $b_{i}^{*}, d_{i}^{*}$ such that

$$
0<a_{1}^{*}<b_{1}^{*}<\frac{k_{1}}{k_{0}} d_{1}^{*}<a_{2}^{*}<b_{2}^{*}<\frac{k_{1}}{k_{0}} d_{2}^{*}<a_{3}^{*}<\cdots<a_{n}^{*}, \quad n \in \mathbb{N},
$$

where $i=1,2, \ldots, n$. In addition, $f$ satisfies the following conditions:

(i) $f\left(t, u_{1}, u_{2}\right) \leq \varphi_{p}\left(\frac{d_{i}^{*}}{L}\right)$ for all $\left(t, u_{1}, u_{2}\right) \in[0,1] \times\left[0, d_{i}^{*}\right] \times\left[-d_{i}^{*}, d_{i}^{*}\right]$;

(ii) $f\left(t, u_{1}, u_{2}\right)>\varphi_{p}\left(\frac{b_{i}^{*}}{N}\right)$ for all $\left(t, u_{1}, u_{2}\right) \in[0,1] \times\left[b_{i}^{*}, d_{i}^{*}\right] \times\left[-d_{i}^{*}, d_{i}^{*}\right]$;

(iii) $f\left(t, u_{1}, u_{2}\right)<\varphi_{p}\left(\frac{a_{i}^{*}}{M}\right)$ for all $\left(t, u_{1}, u_{2}\right) \in[0,1] \times\left[0, a_{i}^{*}\right] \times\left[-d_{i}^{*}, d_{i}^{*}\right]$.

Then the fractional differential equation (2) has at least $2 n-1$ positive solutions.

\section{Examples}

In this section, we present two simple examples to illustrate our theoretical results. In Example 1, it shows the difference between two cases in Section 4.

\section{Example 1 Consider}

$$
\left\{\begin{array}{l}
\left({ }^{C} D^{\frac{8}{3}} u(t)\right)^{\prime}=f\left(t, u(t), u^{\prime}(t)\right), \quad t \in(0,1), \\
k_{0} u(0)-k_{1} u(1)=0, \\
m_{0} u(0)-m_{1} u(1)=0, \\
x^{\prime \prime}(0)=0 .
\end{array}\right.
$$

Case 1: when $f\left(t, u, u^{\prime}\right)$ takes the form as

$$
f\left(t, u, u^{\prime}\right)=u+u^{\prime} \quad \text { for }\left(t, u, u^{\prime}\right) \in[0,1] \times[0, \infty) \times(-\infty,+\infty) .
$$

It is easy to see that

$$
\int_{0}^{t} f\left(s, u, u^{\prime}\right) d s=\int_{0}^{t}\left(u+u^{\prime}\right) d s=t\left(u+u^{\prime}\right) \leq 2 t \max _{t \in[0,1]}\left|u^{\prime}(t)\right|,
$$


then $a_{1}(t)=0$ and $a_{2}(t)=2 t$. Moreover, we see that

$$
\int_{0}^{1} 2 s d s=1<\frac{k_{0}-k_{1}}{k_{0}} \frac{m_{0}-m_{1}}{m_{0}} .
$$

It follows from Theorem 1 that the fractional differential equation (39) has at least one positive solution. However, it is difficult for us to obtain the existence of at least one positive solution to the fractional differential equation (39) by using theorems of the super-linearity and sub-linearity in our paper.

Case 2: when $f\left(t, u, u^{\prime}\right)$ takes the form as

$$
f\left(t, u, u^{\prime}\right)= \begin{cases}-e^{u^{\prime}+1} & \text { for } u^{\prime} \in(-\infty,-1) \\ u^{\prime 5} & \text { for } u^{\prime} \in[-1,1] \\ e^{u^{\prime}-1} & \text { for } u^{\prime} \in(1,+\infty)\end{cases}
$$

By using the continuity of $f$, we obtain that the operator $A$ is completely continuous. It is easy to check that $f_{0}=0$ and $f_{\infty}=\infty$. According to Theorem 3 , we see that fractional differential equation (39) has at least one positive solution. But it is difficult for us to know the existence of positive solution to the fractional differential equation (39) if we use Theorem 1 .

Example 2 Consider

$$
\left\{\begin{array}{l}
\left(\left.{ }^{C} D^{\frac{5}{2}} u(t)\right|^{C} D^{\frac{5}{2}} u(t) \mid\right)^{\prime}=f\left(t, u(t), u^{\prime}(t)\right), \quad t \in(0,1) \\
4 u(0)-u(1)=0 \\
3 u(0)-u(1)=0 \\
x^{\prime \prime}(0)=0
\end{array}\right.
$$

where

$$
f\left(t, u, u^{\prime}\right)= \begin{cases}2 \frac{\Gamma^{2}(3)}{\Gamma^{2}\left(\frac{3}{2}\right)} & \text { for } u \in[0,1] \\ \frac{\Gamma^{2}(3)}{\Gamma^{2}\left(\frac{3}{2}\right)}(57 u-55) & \text { for } u \in(1,2), \\ 59 \frac{\Gamma^{2}(3)}{\Gamma^{2}\left(\frac{3}{2}\right)} & \text { for } u \in[2,+\infty) .\end{cases}
$$

Since $p=3, q=\frac{3}{2}, \alpha=\frac{5}{2}, k_{0}=4, k_{1}=1, m_{0}=3, m_{1}=1, \lambda=1$, a straightforward calculation gives

$$
N=\frac{m_{0}}{m_{0}-m_{1}} \frac{k_{1}}{k_{0}-k_{1}} \frac{\lambda \Gamma(q)}{\Gamma(\alpha+q-1)}=\frac{1}{2} \frac{\Gamma\left(\frac{3}{2}\right)}{\Gamma(3)},
$$

and

$$
L=\frac{k_{0}}{k_{0}-k_{1}} \frac{m_{0}}{m_{0}-m_{1}} \frac{\lambda \Gamma(q)}{\Gamma(\alpha+q-1)}=2 \frac{\Gamma\left(\frac{3}{2}\right)}{\Gamma(3)} .
$$


Taking $a=1, b=2, d=4$ and $c=20$, we see that $0<a<b<d<\frac{1}{4} c$ and

$$
\begin{aligned}
& f\left(t, u, u^{\prime}\right)<\varphi_{3}\left(\frac{a}{L}\right)=4 \frac{\Gamma^{2}(3)}{\Gamma^{2}\left(\frac{3}{2}\right)} \quad \text { for }\left(t, u, u^{\prime}\right) \in[0,1] \times[0,1] \times[-1,1], \\
& f\left(t, u, u^{\prime}\right)>\varphi_{3}\left(\frac{b}{N}\right)=16 \frac{\Gamma^{2}(3)}{\Gamma^{2}\left(\frac{3}{2}\right)} \quad \text { for }\left(t, u, u^{\prime}\right) \in[0,1] \times[2,4] \times[-20,20], \\
& f\left(t, u_{1}, u_{2}\right)<\varphi_{3}\left(\frac{c}{L}\right)=1,600 \frac{\Gamma^{2}(3)}{\Gamma^{2}\left(\frac{3}{2}\right)} \quad \text { for }\left(t, u, u^{\prime}\right) \in[0,1] \times[0,20] \times[-20,20] .
\end{aligned}
$$

By means of Theorem 7, we obtain that the fractional differential equation (40) has at least three distinct positive solutions $u_{1}, u_{2}, u_{3}$ such that

$$
0<\left\|u_{1}\right\|<1, \quad 2<\inf _{t \in[0,1]} u_{2}, \quad 1<u_{3} \quad \text { with } \quad \inf _{t \in[0,1]} u_{3}<2 .
$$

\section{Competing interests}

The authors declare that they have no competing interests.

\section{Authors' contributions}

All authors contributed equally to the manuscript. All authors have read and approved the final version of the manuscript.

\section{Author details}

${ }^{1}$ Department of Mathematics, Xuzhou Institute of Technology, Xuzhou, 221116, China. ${ }^{2}$ Department of Mathematics and Statistics, Qinghai Nationalities University, Qinghai, Xining 810007, China.

\section{Acknowledgements}

This work is supported by Natural Science Foundation of Qinghai Province (No. 2012-Z-910) and Jiangsu Undergraduate Scientific and Technological Innovation Project.

\section{Received: 3 February 2013 Accepted: 11 April 2013 Published: 24 April 2013}

\section{References}

1. Podlubny, I: Fractional Differential Equations. Mathematics in Science and Engineering, vol. 198. Academic Press, New York (1999)

2. Miller, KS, Ross, B: An Introduction to the Fractional Calculus and Fractional Differential Equations. Wiley, New York (1993)

3. Sabatier, J, Agrawal, OP, Machado, JAT: Advances in Fractional Calculus: Theoretical Developments and Applications in Physics and Engineering. Springer, Dordrecht (2007)

4. Glöckle, WG, Nonnenmacher, TF: A fractional calculus approach to self similar protein dynamics. Biophys. J. 68, 46-53 (1995)

5. Metzler, R, Schick, W, Kilian, HG: Nonnenmacher relaxation in filled polymers: a fractional calculus approach. J. Chem. Phys. 103, 7180-7186 (1995)

6. Yakar, A: Initial time difference quasilinearization for Caputo fractional differential equations. Adv. Differ. Equ. 2012,92 (2012)

7. Laskin, N: Fractional Schrödinger equation. Phys. Rev. E 66, 056108-7 (2002)

8. Näsholm, SP, Holm, S: Linking multiple relaxation, power-law attenuation, and fractional wave equations. J. Acoust. Soc. Am. 130, 3038-3045 (2011)

9. Chang, YK, Nieto, JJ: Some new existence results for fractional differential inclusions with boundary conditions. Math. Comput. Model. 49(3-4), 605-609 (2009)

10. Bai, ZB, Lü, H: Positive solutions for boundary value problem of nonlinear fractional differential equation. J. Math. Anal. Appl. 311, 495-505 (2005)

11. Erturk, VS, Momani, S: Solving systems of fractional differential equations using differential transform method. J. Comput. Appl. Math. 215, 142-151 (2008)

12. Kilbas, AA, Trujillo, JJ: Differential equations of fractional order: methods, results and problems - I. Appl. Anal. 78 153-192 (2001)

13. Lakshmikantham, V, Leela, S, Vasundhara Devi, J: Theory of Fractional Dynamic Systems. Cambridge Academic Publishers, Cambridge (2009)

14. Baleanu, D, Mustafa, OG, O'Regan, D: On a fractional differential equation with infinitely many solutions. Adv. Differ. Equ. 2012, 145 (2012)

15. Su, YH, Feng, Z: Existence theory for an arbitrary order fractional differential equation with deviating argument. Acta Appl. Math. 118, 81-105 (2012) 
16. Han, X, Gao, H: Existence of positive solutions for eigenvalue problem of nonlinear fractional differential equations. Adv. Differ. Equ. 2012, 66 (2012)

17. Leibenson, LS: General problem of the movement of a compressible fluid in a porous medium. Izv. Akad. Nauk Kirg. SSSR 9, 7-10 (1983) (in Russian)

18. Cheng, C, Su, YH, Feng, Z: Multiple positive solutions of fractional differential equations with derivative terms. Electron. J. Differ. Equ. 2012, 215 (2012)

19. Sudsutad, W, Tariboon, J: Boundary value problems for fractional differential equations with three-point fractional integral boundary conditions. Adv. Differ. Equ. 2012, 93 (2012)

20. Krasnosel'skii, M: Positive Solutions of Operator Equations. Noordhoff, Groningen (1964)

21. Agarwal, RP, Meehan, M, O'Regan, D: Fixed Point Theory and Applications. Cambridge University Press, Cambridge (2001)

22. Leggett, RW, Williams, LR: Multiple positive fixed points of nonlinear operators on ordered Banach spaces. Indiana Univ. Math. J. 28, 673-688 (1979)

23. Avery, Rl, Henderson, J: Two positive fixed points of nonlinear operator on ordered Banach spaces. Commun. Appl. Nonlinear Anal. 8, 27-36 (2001)

24. Avery, Rl, Peterson, A: Three positive fixed points of nonlinear operators on ordered Banach spaces. Comput. Math. Appl. 42, 313-422 (2001)

doi:10.1186/1687-1847-2013-119

Cite this article as: Su et al.: Existence criteria for positive solutions of $p$-Laplacian fractional differential equations with derivative terms. Advances in Difference Equations 2013 2013:119.

\section{Submit your manuscript to a SpringerOpen ${ }^{\circ}$ journal and benefit from:}

- Convenient online submission

- Rigorous peer review

- Immediate publication on acceptance

- Open access: articles freely available online

- High visibility within the field

- Retaining the copyright to your article 